\title{
Non-equilibrium effects on flow past a circular cylinder in the slip and early transition regime
}

\author{
Xiao-Jun Gu ${ }^{1, \dagger}$, Robert W. Barber ${ }^{1}$, Benzi John ${ }^{1}$ and David R. Emerson ${ }^{1}$ \\ ${ }^{1}$ Scientific Computing Department, STFC Daresbury Laboratory, Warrington WA4 4AD, UK
}

(Received 11 June 2018; revised 17 October 2018; accepted 24 October 2018; first published online 10 December 2018)

This paper presents a comprehensive investigation into flow past a circular cylinder where compressibility and rarefaction effects play an important role. The study focuses on steady subsonic flow in the Reynolds-number range 0.1-45. Rarefaction, or non-equilibrium, effects in the slip and early transition regime are accounted for using the method of moments and results are compared to data from kinetic theory obtained from the direct simulation Monte Carlo method. Solutions obtained for incompressible continuum flow serve as a baseline to examine non-equilibrium effects on the flow features. For creeping flow, where the Reynolds number is less than unity, the drag coefficient predicted by the moment equations is in good agreement with kinetic theory for Knudsen numbers less than one. When flow separation occurs, we show that the effects of rarefaction and velocity slip delay flow separation and will reduce the size of the vortices downstream of the cylinder. When the Knudsen number is above 0.028, the vortex length shows an initial increase with the Reynolds number, as observed in the standard no-slip continuum regime. However, once the Reynolds number exceeds a critical value, the size of the downstream vortices decreases with increasing Reynolds number until they disappear. An existence criterion, which identifies the limits for the presence of the vortices, is proposed. The flow physics around the cylinder is further analysed in terms of velocity slip, pressure and skin friction coefficients, which highlights that viscous, rarefaction and compressibility effects all play a complex role. We also show that the local Knudsen number, which indicates the state of the gas around the cylinder, can differ significantly from its free-stream value and it is essential that computational studies of subsonic gas flows in the slip and early transition regime are able to account for these strong non-equilibrium effects.

Key words: compressible flows, rarefied gas flow, vortex flows

\section{Introduction}

Flow past a stationary circular cylinder is a classical problem in fluid mechanics. Although the geometric configuration is relatively simple, the physics associated

$\dagger$ Email address for correspondence: xiaojun.gu@stfc.ac.uk 
with the flow around the cylinder exhibits a range of important phenomena, such as flow separation with attached eddies and the formation of the well-known vortex street. Consequently, the problem has attracted much attention involving theoretical, experimental and computational investigations. Early theoretical work was concerned with uniform flow past a circular cylinder and focused on the steady, low-speed viscous flow of an incompressible fluid where the flow characteristics depend solely on the Reynolds number, Re. These theoretical studies, which were focused on low-Reynolds-number or 'creeping flow' solutions, where $R e \ll 1$, can be traced back to the pioneering work of Stokes (1851). In his work, Stokes neglected the inertia terms and reformulated the Stokes equation in the form $\nabla^{4} \psi=0$ to derive a solution, where $\psi$ is the streamfunction (Basset 1888; Lamb 1911; Bairstow, Cave \& Lang 1922; Apelt 1961; Happel \& Brenner 1983). Although this approach works for a sphere, if the steady motion of a cylinder is considered, not all conditions can be satisfied and this led to the famous Stokes paradox, where a steady solution to the problem is not possible (Basset 1888). Oseen (1910) resolved the paradox by noting that the inertial terms need to be taken into account and proposed a linearised set of equations that partially accounted for the inertial terms. Lamb (1911) subsequently developed a solution to Oseen's equations that was valid for small Re. Following the work of Oseen and Lamb, a series of asymptotic analyses of different orders were developed (Kaplun 1957; Proudman \& Pearson 1957; Underwood 1969; Skinner 1975; Keller \& Ward 1996). Despite significant progress in developing theoretical solutions to the Navier-Stokes equations, it has proved extremely challenging and results are generally limited to low-Reynolds-number flows.

As noted by Tritton (1988), when $R e \ll 1$ the flow around the cylinder is symmetric, both upstream and downstream. However, as the Reynolds number increases beyond unity and exceeds some critical value, $R e_{\text {onset }}$, which is around 3-7 (Sen, Mittal \& Biswas 2009), the fluid undergoes a steady separation and forms an attached pair of symmetric contra-rotating vortices at the base of the cylinder. The fluid in these vortices circulates continuously, not moving downstream. The length of these eddies will grow approximately linearly with increasing $R e$ until they reach a second critical value, $R e_{c}$, around 40-50 (Kumar \& Mittal 2006), where the wake downstream of the cylinder becomes unsteady (Zdravkovich 1997). Experiments have naturally played a crucial part in identifying and understanding the various flow regimes that exist, particularly in trying to establish values for $R e_{\text {onset }}$ and $R e_{c}$, and especially for unsteady flows at higher Reynolds numbers. Strouhal (1878) completed some of the earliest observations showing that the frequency of oscillation is linked to the fluid velocity. Another important quantity for flow past a circular cylinder is the drag coefficient, $C_{D}$. It has been experimentally investigated extensively, along with the wake geometry, for incompressible flow in the continuum regime (Taneda 1956; Tritton 1959; Acrivos et al. 1968; Coutanceau \& Bouard 1977; Huner \& Hussey 1977; Wu et al. 2004). With the advance of computers in the 1960s, the numerical solution of the Navier-Stokes equations for flow past a cylinder became possible, allowing researchers to study the details of the flow physics (Son \& Hanratty 1969; Dennis \& Chang 1970; Fornberg 1980; Sen et al. 2009). By analysing the numerical solution of the incompressible Navier-Stokes equations with direct time integration and linear stability analysis methods, Kumar \& Mittal (2006) obtained a value for the second critical Reynolds number, $R e_{c}$, that is just above 47 .

The vast majority of research studying flow past a circular cylinder has been for conventional fluid mechanics where the velocity at the cylinder surface is zero, i.e. the classic no-slip boundary condition. However, when the gas is in a non-equilibrium or 
rarefied state, the no-slip assumption is no longer valid and both the Reynolds number and the Knudsen number are required to determine the flow physics. The Knudsen number, $K n$, relates the ratio of the molecular mean free path of the gas, $\lambda$, to a characteristic length of the geometry, e.g. the diameter of a cylinder, $D$, and provides a convenient way to determine the extent of the non-equilibrium or rarefaction of the gas. Conventionally, when $K n \lesssim 0.001$, the traditional Navier-Stokes equations are valid and the no-slip boundary condition can be used. When $0.001 \lesssim K n \lesssim 0.1$, the flow is in the slip regime and the Navier-Stokes-Fourier (NSF) equations, coupled with appropriate velocity-slip and temperature-jump wall boundary conditions, are able to predict certain main features of the flow for simple problems, but care is needed when thermal effects are present (Sone 2000; John, Gu \& Emerson 2010). If the Knudsen number lies in the range $0.1 \lesssim K n \lesssim 10$, the flow is in the transition regime and the NSF equations are no longer valid. For $K n \gtrsim 10$, the gas is in the free-molecular or collisionless flow regime and kinetic theory is required to study the flow physics. Solutions of the Boltzmann equation (Cercignani 1975, 2000) are required and the de facto standard for simulating gas flow in the transition regime and beyond is the direct simulation Monte Carlo (DSMC) method (Bird 1994). It is important to note that the Knudsen number is proportional to the product of the Mach number and Reynolds number and is discussed further in $\S 4$.

Despite the numerous studies of flow past a circular cylinder in the continuum regime, for both compressible and incompressible flows, studies involving rarefied flows remain relatively scarce in the literature. In contrast to the difficulty in deriving an analytical solution to flow past a cylinder for the Navier-Stokes equations, exact solutions for drag in the free-molecular regime, as well as other aerodynamic forces, have been derived for many objects. These include a flat plate at an angle of attack (Tsien 1946; Schaaf \& Chambre 1961; Sentman 1961), flow past a stationary sphere (Epstein 1924) and the circular cylinder (Heineman 1948; Ashley 1949; Stalder, Goodwin \& Creager 1951; Schaaf \& Chambre 1961). There have been several attempts to extend continuum-based analytical expressions for drag; for example, if an analytical solution is available for the Navier-Stokes equations, then it is possible to include the effects of velocity slip, provided geometrical effects like curvature are properly accounted for (Barber et al. 2004; Lockerby et al. 2004). In the case of a sphere, Basset (1888) extended the solution developed by Stokes to include velocity slip. For the cylinder, Pich (1969) derived an expression for $C_{D}$ from the early transition to the free-molecular regime by defining a 'molecular layer' around the cylinder and considering Lamb's classical hydrodynamic approximation away from the cylinder. Yamamoto \& Sera (1985) studied low-Reynolds-number flow past a cylinder using the linearised Bhatnagar-Gross-Krook (BGK) kinetic equation. Westerkamp \& Torrilhon (2012) derived an analytic solution for creeping flow past a cylinder based on a linearised version of the regularised 13 moment equations. Although beyond the scope of the current paper, there has been interest in understanding high-speed rarefied gas flow past a cylinder. Experimental investigations have ranged from the continuum to free-molecular flow (Maslach \& Schaaf 1963; Ponomarev \& Filippova 1969), whereas simulations based on kinetic approaches have recently been used ( $\mathrm{Li}$ et al. 2011; John et al. 2016; Volkov \& Sharipov 2017).

It is clear that there is little data available when $1<R e<R e_{c}$ and the gas is in the slip and early transition regime. Indeed, little is known about low-Reynolds-number compressible flow, although interest is steadily growing due to the possibility of flight on Mars to survey the terrain (Munday et al. 2015). In contrast to the Earth, the atmosphere on Mars is quite rarefied. Recently, Canuto \& Taira (2015) performed 
direct numerical simulation of flow past a cylinder with moderate Reynolds and Mach numbers using the compressible Navier-Stokes equations. Their flow conditions lay in the range $0<K n \leqslant 0.037$ but rarefaction effects were not considered, which could result in an overprediction of both $C_{D}$ and the vortex size. Hu, Sun \& Fan (2009) computed the drag coefficient in the non-equilibrium flow regime with a hybrid kinetic/continuum approach and found that there is a complex interplay between rarefaction and compressibility effects. Currently, there is a lack of fundamental studies in low-Reynolds-number compressible flows and particularly for problems where non-equilibrium effects are important.

Extending hydrothermodynamics into the slip and transition regimes is one of the most promising approaches for accurately capturing the physics associated with non-equilibrium gas flows (Muller \& Ruggeri 1993; Struchtrup 2005). The method of moments, originally proposed by Grad (1949), provides an approximate solution procedure to the Boltzmann equation and is under active development to bridge the gap between hydrothermodynamics and kinetic theory. In this approach, the Boltzmann equation is satisfied in a certain average sense rather than at the molecular distribution function level. How many moments are required largely depends on the flow regime. It was found (Gu, Emerson \& Tang 2010; Young 2011; Gu \& Emerson 2014) that the regularised 13 moment equations (R13) are not adequate enough to capture the Knudsen layer in Kramers' problem and the regularised 26 moment equations (R26) are required to accurately reproduce the velocity defect found with kinetic data. However, both the R13 and R26 equations are able to capture many of the non-equilibrium phenomena observed using kinetic theory. These include effects such as the tangential heat flux in planar Couette flow and the bimodal temperature profile in planar force-driven Poiseuille flow (Gu \& Emerson 2007, 2009; Taheri \& Struchtrup 2009; Taheri, Torrilhon \& Struchtrup 2009). With several extra macroscopic governing equations, the moment method is slightly more expensive than the NSF equations to solve numerically. However, it is much more computationally efficient than the kinetic approaches and capable of capturing the non-equilibrium effects with a high degree of accuracy in the slip and early transition regime.

In the present study, we investigate the impact of rarefaction on steady subsonic compressible flow past a circular cylinder in the Reynolds-number range $0.1 \leqslant R e<$ 45 , i.e. before the onset of classic unsteady vortex shedding. To capture the nonequilibrium effects, we use the method of moments, as described in $\S 2$, and compare our results with kinetic theory using DSMC, which is outlined in $\S 3$. The problem formulation is described in $\S 4$ and the computed results for drag, the onset of flow separation, the location of the separation point and the size of the attached eddies will be examined in detail in $\S 5$ in terms of Reynolds number, Knudsen number as well as Mach number. Our findings are discussed in $\S 6$.

\section{An overview of the method of moments}

The traditional hydrodynamic quantities of density $\rho$, velocity $u_{i}$ and temperature $T$ correspond to the first five lowest-order moments of the molecular distribution function, $f$. The governing equations of these hydrodynamic quantities for a dilute gas can be obtained from the Boltzmann equation and represent mass, momentum and energy conservation laws, respectively (Struchtrup 2005):

$$
\frac{\partial \rho}{\partial t}+\frac{\partial \rho u_{i}}{\partial x_{i}}=0,
$$




$$
\frac{\partial \rho u_{i}}{\partial t}+\frac{\partial \rho u_{i} u_{j}}{\partial x_{j}}+\frac{\partial \sigma_{i j}}{\partial x_{j}}=-\frac{\partial p}{\partial x_{i}}
$$

and

$$
\frac{\partial \rho T}{\partial t}+\frac{\partial \rho u_{i} T}{\partial x_{i}}+\frac{2}{3 R} \frac{\partial q_{i}}{\partial x_{i}}=-\frac{2}{3 R}\left(p \frac{\partial u_{i}}{\partial x_{i}}+\sigma_{i j} \frac{\partial u_{j}}{\partial x_{i}}\right) .
$$

Here $t$ and $x_{i}$ are temporal and spatial coordinates, respectively, and any suffix $i$, $j$ or $k$ represents the usual summation convention. The pressure $p$ is related to the temperature and density by the ideal gas law, $p=\rho R T$, where $R$ is the specific gas constant. However, the stress term, $\sigma_{i j}$, and heat flux term, $q_{i}$, given in (2.2) and (2.3) are unknown. The classical way to close this set of equations is through a Chapman-Enskog expansion (see Chapman \& Cowling 1970) of the molecular distribution function, $f$. When $f$ is truncated at the first order of $K n$, the gradient transport mechanism is obtained as

$$
\sigma_{i j}=\sigma_{i j}^{N S F}=-\frac{2 \mu}{A_{\sigma}} \frac{\partial u_{\langle i}}{\partial x_{j\rangle}} \quad \text { and } \quad q_{i}=q_{i}^{N S F}=-\frac{5 \mu R}{2 A_{q}} \frac{\partial T}{\partial x_{i}},
$$

and equations (2.1)-(2.3) become the NSF equations. The angular brackets are used to denote the traceless part of a symmetric tensor. Alternatively, the governing equations of $\sigma_{i j}$ and $q_{i}$ can be derived from the Boltzmann equation, as proposed by Grad (1949):

$$
\frac{\partial \sigma_{i j}}{\partial t}+\frac{\partial u_{k} \sigma_{i j}}{\partial x_{k}}+\frac{\partial m_{i j k}}{\partial x_{k}}=-A_{\sigma} \frac{p}{\mu} \sigma_{i j}-2 p \frac{\partial u_{\langle i}}{\partial x_{j\rangle}}-\frac{4}{5} \frac{\partial q_{\langle i}}{\partial x_{j\rangle}}-2 \sigma_{k\langle i} \frac{\partial u_{j\rangle}}{\partial x_{k}}
$$

and

$$
\begin{aligned}
\frac{\partial q_{i}}{\partial t}+\frac{\partial u_{j} q_{i}}{\partial x_{j}}+\frac{1}{2} \frac{\partial R_{i j}}{\partial x_{j}}= & \frac{-A_{q} \frac{p}{\mu} q_{i}-\frac{5}{2} p R \frac{\partial T}{\partial x_{i}}-\frac{7 \sigma_{i k} R}{2} \frac{\partial T}{\partial x_{k}}}{}-R T \frac{\partial \sigma_{i k}}{\partial x_{k}}+\frac{\sigma_{i j}}{\rho}\left(\frac{\partial p}{\partial x_{j}}+\frac{\partial \sigma_{j k}}{\partial x_{k}}\right) \\
& -\frac{2}{5}\left(\frac{7}{2} q_{k} \frac{\partial u_{i}}{\partial x_{k}}+q_{k} \frac{\partial u_{k}}{\partial x_{i}}+q_{i} \frac{\partial u_{k}}{\partial x_{k}}\right)-\frac{1}{6} \frac{\partial \Delta}{\partial x_{i}}-m_{i j k} \frac{\partial u_{j}}{\partial x_{k}} .
\end{aligned}
$$

By applying a first-order Chapman-Enskog expansion to equations (2.5) and (2.6), only the underlined terms are retained. Therefore, they reduce to equation (2.4) and the NSF equations are recovered. The higher moments $m_{i j k}, R_{i j}$ and $\Delta$ need to be known to obtain a solution of equations (2.1)-(2.3), (2.5) and (2.6). Their algebraic expressions, in terms of the derivatives of lower moments, can be used to close this set of equations, as in the R13 equation approach (Struchtrup \& Torrilhon 2003). Alternatively, the governing equations of $m_{i j k}, R_{i j}$ and $\Delta$ derived from the Boltzmann equation can be used to provide information required in equations (2.5) and (2.6). They are (Gu \& Emerson 2009):

$$
\begin{gathered}
\frac{\partial m_{i j k}}{\partial t}+\frac{\partial u_{l} m_{i j k}}{\partial x_{l}}+\frac{\partial \phi_{i j k l}}{\partial x_{l}}=-A_{m} \frac{p}{\mu} m_{i j k}-3 R T \frac{\partial \sigma_{\langle i j}}{\partial x_{k\rangle}}-\frac{3}{7} \frac{\partial R_{\langle i j}}{\partial x_{k\rangle}}+\mathfrak{M}_{i j k}, \\
\frac{\partial R_{i j}}{\partial t}+\frac{\partial u_{k} R_{i j}}{\partial x_{k}}+\frac{\partial \psi_{i j k}}{\partial x_{k}}=-A_{R 1} \frac{p}{\mu} R_{i j}-\frac{28}{5} R T \frac{\partial q_{\langle i}}{\partial x_{j\rangle}}-2 R T \frac{\partial m_{i j k}}{\partial x_{k}}-\frac{2}{5} \frac{\partial \Omega_{\langle i}}{\partial x_{j\rangle}}+\Re_{i j}
\end{gathered}
$$




$$
\begin{array}{cccccccccc}
A_{\sigma} & A_{q} & A_{m} & A_{R 1} & A_{R 2} & A_{\Delta 1} & A_{\Delta 2} & A_{\phi} & A_{\psi} & A_{\Omega} \\
1.0 & 2 / 3 & 3 / 2 & 7 / 6 & 2 / 3 & 2 / 3 & 2 / 3 & 2.097 & 1.698 & 1.0
\end{array}
$$

TABLE 1. Collision constants in the moment equations for Maxwell molecules.

and

$$
\frac{\partial \Delta}{\partial t}+\frac{\partial \Delta u_{i}}{\partial x_{i}}+\frac{\partial \Omega_{i}}{\partial x_{i}}=-A_{\Delta 1} \frac{p}{\mu} \Delta-8 R T \frac{\partial q_{k}}{\partial x_{k}}+\aleph,
$$

in which $\mathfrak{M}_{i j k}, \mathfrak{R}_{i j}$ and $\aleph$ are the nonlinear source terms listed in appendix A. In equations (2.7)-(2.9), higher-order unknown moments $\phi_{i j k l}, \quad \psi_{i j k}$ and $\Omega_{i}$ are introduced into the system of governing equations, which can be obtained from the Boltzmann equation. However, even higher-order moments will appear in their equations. This is the closure problem in the moment method. Alternatively, they can be approximated by algebraic expressions in terms of the derivatives of lower moments. In the R26 equations ( $\mathrm{Gu} \&$ Emerson 2009), they were obtained by a Chapman-Enskog expansion. For convenience, they can be expressed as gradient transport terms and high-order nonlinear terms, respectively, by

$$
\begin{aligned}
\phi_{i j k l} & =-\frac{4 \mu}{A_{\phi} \rho} \frac{\partial m_{\langle i j k}}{\partial x_{l\rangle}}+\phi_{i j k l}^{N L}, \\
\psi_{i j k} & =-\frac{27 \mu}{7 A_{\psi} \rho} \frac{\partial R_{\langle i j}}{\partial x_{k\rangle}}+\psi_{i j k}^{N L}
\end{aligned}
$$

and

$$
\Omega_{i}=-\frac{7}{3} \frac{\mu}{A_{\Omega} \rho} \frac{\partial \Delta}{\partial x_{i}}-4 \frac{\mu}{A_{\Omega} \rho} \frac{\partial R_{i k}}{\partial x_{k}}+\Omega_{i}^{N L} .
$$

The full expressions of the nonlinear terms $\phi_{i j k l}^{N L}, \quad \psi_{i j k}^{N L}$ and $\Omega_{i}^{N L}$ are provided by $\mathrm{Gu}$ $\&$ Emerson (2009). The values of the collision constants, $A_{\sigma}, A_{q}, A_{m}, A_{R 1}, A_{R 2}, A_{\Delta 1}$, $A_{\Delta 2}, A_{\phi}, A_{\psi}$ and $A_{\Omega}$, depend on the molecular collision model adopted and represent the relaxation time scale for each moment. They are given in table 1 for the case of Maxwell molecules (Truesdell \& Muncaster 1980; Struchtrup 2005), as employed in the present study. Although a dilute monatomic gas is employed, all the findings in the present study have relevance to realistic gases, such as air.

To apply any of the foregoing models to flows in confined geometries, appropriate wall boundary conditions are required to determine a unique solution. $\mathrm{Gu} \&$ Emerson (2009) obtained a set of wall boundary conditions for the R26 equations based on Maxwell's kinetic wall boundary condition (Maxwell 1879) and a fifth-order approximation of the molecular distribution function in Hermite polynomials. In a frame where the coordinates are attached to the wall, with $n_{i}$ the normal vector out of the wall pointing towards the gas and $\tau_{i}$ the tangential vector of the wall, the slip velocity parallel to the wall, $u_{\tau}$, and temperature-jump conditions are

$$
u_{\tau}=-\frac{2-\alpha}{\alpha} \sqrt{\frac{\pi R T}{2}} \frac{\sigma_{n \tau}}{p_{\alpha}}-\frac{q_{\tau}}{5 p_{\alpha}}-\frac{m_{n n \tau}}{2 p_{\alpha}}+\frac{9 \Omega_{\tau}+70 \psi_{n n \tau}}{2520 p_{\alpha} R T}
$$

and

$$
R T-R T_{w}=-\frac{2-\alpha}{\alpha} \sqrt{\frac{\pi R T}{2}} \frac{q_{n}}{2 p_{\alpha}}-\frac{R T \sigma_{n n}}{4 p_{\alpha}}+\frac{u_{\tau}^{2}}{4}-\frac{75 R_{n n}+28 \Delta}{840 p_{\alpha}}+\frac{\phi_{n n n n}}{24 p_{\alpha}},
$$


where

$$
p_{\alpha}=p+\frac{\sigma_{n n}}{2}-\frac{30 R_{n n}+7 \Delta}{840 R T}-\frac{\phi_{n n n n}}{24 R T} .
$$

Here $\sigma_{n n}, \sigma_{n \tau}, q_{\tau}, m_{n n \tau}, m_{n n n}, R_{n n}, \psi_{n n \tau}, \Omega_{\tau}$ and $\phi_{n n n n}$ are the tangential and normal components of $\sigma_{i j}, q_{i}, m_{i j k}, R_{i j}, \psi_{i j k}, \Omega_{i}$ and $\phi_{i j k l}$ relative to the wall, respectively. It should be noted that the normal velocity at the wall $u_{n}=0$, since there is no gas flow through the wall. The accommodation coefficient, $\alpha$, represents the fraction of gas molecules that will be diffusively reflected with a Maxwellian distribution at the temperature of the wall, $T_{w}$. The remaining fraction $(1-\alpha)$ of gas molecules will undergo specular reflection. The rest of the wall boundary conditions are listed in appendix $\mathrm{C}$ of $\mathrm{Gu} \&$ Emerson (2009). Equations (2.13) and (2.14) are similar to the velocity-slip and temperature-jump conditions for the NSF equations (Cercignani 1975; Gad-el-Hak 1999) with the additional underlined terms on the right-hand side providing the higher-order moment contributions which are not available in the NSF model. However, these higher-order moment terms can be used to derive a second-order slip boundary condition for the NSF equations (Taheri \& Struchtrup 2010). The solution of the NSF equations in the present study is associated with the wall boundary conditions (2.13) and (2.14) without the underlined terms.

A pressure-based numerical algorithm was introduced by $\mathrm{Gu} \&$ Emerson (2007, 2009) to solve the moment equations for weakly compressible and low-speed flows. It has been successfully applied in the study of pressure-driven Poiseuille flow (Tang et al. 2013), thermal transpiration flow (Sheng et al. 2014) and gas flows in porous media (Lu et al. 2017; Wu et al. 2017). To accommodate the compressibility of the gas flow in the present study, the pressure-based method (Ferziger \& Perić 2002) for arbitrary Mach number has been employed. In addition to the pressure correction, a density correction is integrated into the derivation of the pressure correction equation. The resultant pressure correction equation is no longer a discretised Poisson equation. It contains both convective and unsteady terms. When the Mach number is not negligibly small, the convective term dominates. The pressure correction method automatically adjusts to the local nature of the flow and is ideal for the purpose of the present study.

\section{Description of the direct simulation Monte Carlo method}

The DSMC method is based on the discrete molecular nature of a gas and essentially captures the physics of a dilute gas governed by the Boltzmann equation. The DSMC method was first introduced by Bird in the early 1960s (Bird 1963) as a technique to analyse high-Knudsen-number flows, primarily targeted towards aerospace applications. Since then, the DSMC method has evolved into a powerful numerical approach and is considered one of the most reliable methods for simulating non-equilibrium gaseous flows. However, it is very computationally expensive, particularly for low-Reynolds-number flows in the early transition regime. Despite significant efforts to overcome the numerical difficulties and computing costs, solutions using the Boltzmann equation or DSMC are still too difficult to be widely used in practical engineering applications. Variants of DSMC, like the LVDSMC method (Baker \& Hadjiconstantinou 2005), employ variance reduction techniques to reduce the statistical noise. However, they are more suited for problems involving extremely low flow speeds or very small thermal gradients. This has led to alternative approaches being developed, as exemplified by the method of moments, which aim to 
alleviate the computational difficulties associated with kinetic theory but are accurate enough for engineering design.

As the most reliable method for non-equilibrium gaseous flow simulations, the DSMC method is employed to complement the computational solution of the macroscopic equations. A detailed explanation of the steps involved in the DSMC method can be found in Bird (1963). In contrast to the molecular dynamics (MD) method (Allen \& Tildesley 1987), which is deterministic, the exact trajectory of each particle is not calculated in DSMC. Instead, a stochastic algorithm is used to evaluate the collision probabilities and scattering distributions based on kinetic theory. Although the actual nature of the molecular interactions is complex, they can be treated in a DSMC simulation through the use of phenomenological collision models.

The DSMC simulations in this work have been carried out using SPARTA (Gallis et al. 2014). The software is a highly scalable parallel open-source DSMC code recently developed by Sandia National Laboratory (Plimpton \& Gallis 2017). The code has been validated on several benchmark test cases (Gallis et al. 2016, 2017) and is widely used by the DSMC community. For all DSMC simulations in this study, the gas was assumed to be argon and the variable hard sphere (VHS) model was employed. Previous theoretical and numerical studies ( $\mathrm{Gu} \&$ Emerson 2009, 2011, 2014; Gu et al. 2010; Gu, Zhang \& Emerson 2016) have indicated that the results from the R26 equations using Maxwell molecules and the DSMC simulations using a VHS model are in close agreement with each other. Bird's no-time-counter (NTC) scheme (Bird 1994) has been employed for calculating the collision probabilities in a cell. Particle-wall interactions were assumed to be fully diffuse. Additionally, the well-known guidelines have been followed with respect to the cell size, time step and particle numbers that are needed for accurate DSMC calculations. The cell sizes in our study are defined to be smaller than one-third of the mean free path. Accordingly, the total number of cells in our simulations ranges from a few hundred thousand to several hundred million, depending on the Knudsen number and operating pressure under consideration. The time step for the DSMC simulations is taken to be five times smaller than $\Delta x_{\min } /\left(V_{m p}+U_{\infty}\right)$, where $\Delta x_{\text {min }}$ is the smallest cell dimension, $V_{m p}$ is the most probable molecular velocity given by $V_{m p}=\sqrt{2 R T_{\infty}}$, and $U_{\infty}$ is the free-stream velocity. To minimise statistical noise, an average of at least a hundred particles per cell has been considered and the sampling phase has been carried out over a period of several hundred thousand time steps.

The DSMC computational domain is a square shape, with the cylinder centrally placed. The inflow and outflow boundary conditions in our DSMC simulations are implemented by injecting particles based on the Maxwellian distribution and corresponding to the flow conditions at the boundary. For the inflow boundary case, particles are injected based on the free-stream velocity and density conditions. At the subsonic outflow, particles can enter the domain from outside, the properties of which are predominantly determined by the interior solution. We have followed the characteristic boundary conditions (Hirsch 1991; Sun \& Boyd 2004; John et al. 2016) for the subsonic outflow, based on which the exit pressure is specified while other properties like velocity and density are derived from values extrapolated from the adjacent cell in the interior domain. The spatial extent of the DSMC computational domain required for an accurate simulation depends on the Reynolds number and Knudsen number under consideration. In general, a larger computational domain size is required for cases with low Reynolds numbers when compared to cases with high flow velocities (high Reynolds numbers). Accordingly, the largest computational domain size considered is $60 D \times 60 D$, corresponding to the case with $R e=0.5$ 
and $K n_{\infty}=0.2$, whereas the smallest computational domain size is $15 D \times 15 D$, corresponding to the case with $R e=26$ and $K n_{\infty}=0.07$.

A major limitation of the DSMC method is that, although it is valid for all flow regimes, the technique becomes computationally prohibitive in the near-continuum regime. Additionally, statistical noise becomes increasingly significant for very low-speed flows, requiring excessively large sampling periods to improve the signal-to-noise ratio. Therefore, we have carried out DSMC computations of only a few selected combinations of Reynolds number and Knudsen number, for which the simulations were relatively computationally affordable. The simulation time for the DSMC cases that we considered in the present study varies depending on the operating flow conditions. However, in general, DSMC requires 30-50 times more $\mathrm{CPU}$ time than for solving the macroscopic equations.

\section{Problem formulation}

Gaseous flow past a circular cylinder with a uniform wall temperature, $T_{w}$, is computationally studied using the moment equations and the DSMC method. The computational domain for the macroscopic equations is illustrated in figure 1 with a circular cylinder of diameter $D$. A gas with a free-stream velocity $u_{\infty}$, temperature $T_{\infty}$ and pressure $p_{\infty}$ (or density $\rho_{\infty}$ ) flows towards the cylinder. The origin of the Cartesian coordinates $(x, y)$ and the polar coordinates $(r, \theta)$ sits at the centre of the circular cylinder. The ratio of the computational domain length, $L$, to the width, $H$, is fixed at 10 , while the aspect ratio $H / D$ varies for different Reynolds numbers. The diameter of the cylinder, $D$, is chosen as the characteristic length, so that the Reynolds number, $R e$, is calculated from

$$
R e=\frac{\rho_{\infty} u_{\infty} D}{\mu_{\infty}},
$$

where $\mu_{\infty}$ is the gas viscosity at temperature $T_{\infty}$. The Knudsen number, $K n_{\infty}$, is based on the state of the free stream and is obtained from

$$
K n_{\infty}=\frac{\lambda_{\infty}}{D}
$$

in which $\lambda_{\infty}$ is the mean free path of the gaseous free stream defined by

$$
\lambda_{\infty}=\frac{\mu_{\infty}}{p_{\infty}} \sqrt{\frac{\pi R T_{\infty}}{2}},
$$

which indicates that, when the pressure of a gas is lower, the mean free path of the gas becomes larger and vice versa.

For ideal gases, the Mach number of the incoming stream, $M a_{\infty}$, is estimated from

$$
M a_{\infty}=\frac{u_{\infty}}{\sqrt{\gamma R T_{\infty}}}
$$

where $\gamma$ is the ratio of specific heat capacities. For the monatomic gas in the present study, $\gamma=5 / 3$. The relationship between $R e, K n_{\infty}$ and $M a_{\infty}$ is readily obtained as

$$
M a_{\infty}=\sqrt{\frac{2}{\gamma \pi}} \operatorname{Re} K n_{\infty} .
$$




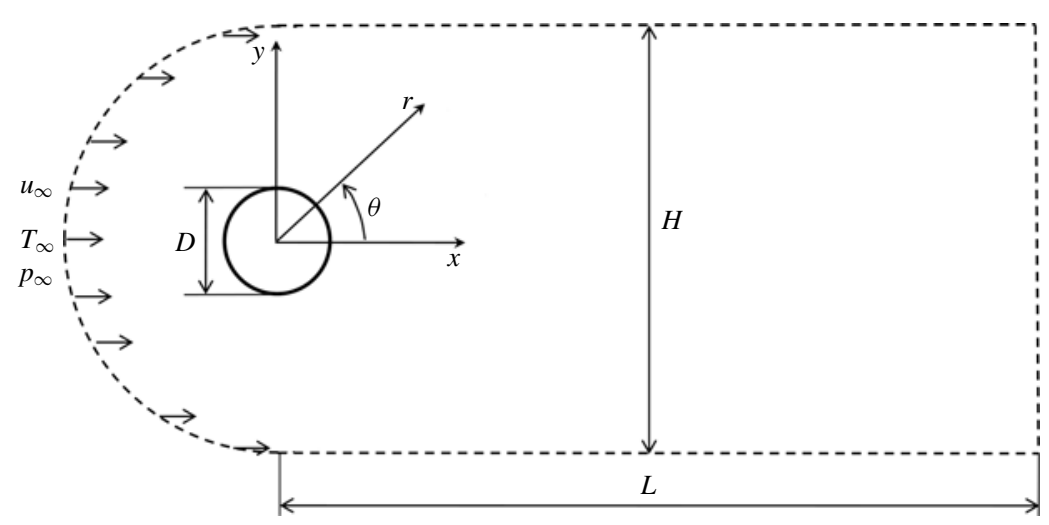

FIGURE 1. Schematic of the macroscopic equation computational domain for the gas flow past a circular cylinder.

It can be seen from (4.5) that $M a_{\infty}$ is proportional to the product of $R e$ and $K n_{\infty}$. When the gas departs from the continuum state, i.e. $K n \gg 0$, the Reynolds-number effect on the compressibility of the gas increases significantly. In the present study, the investigations are limited to subsonic $\left(M a_{\infty}<1\right)$, creeping and steady separation $(R e<45)$ flows in the slip and early transition regime $\left(K n_{\infty}<1\right)$.

\section{Results and discussion}

Macroscopic sets of the NSF, R13 and R26 equations are solved numerically for flows past a stationary cylinder with a wide range of Reynolds and Knudsen numbers determined by the free-stream parameters, $u_{\infty}, T_{\infty}$ and $p_{\infty}$. The Dirichlet boundary condition is used for the side boundaries and the Neumann boundary condition for the downstream boundary, as illustrated in figure 1. For convenience, the cylinder wall temperature, $T_{w}$, is set to the free-stream temperature, $T_{\infty}$. A fully diffusive cylinder surface is assumed, i.e. the accommodation coefficient, $\alpha=1$, and a body-fitted mesh is employed around the cylinder. The viscosity was obtained from Sutherland's law (White 1991). The computed results are analysed in terms of the drag coefficient, $C_{D}$, the onset of the twin vortices downstream of the cylinder characterised in terms of the vortex length $l$, defined as the distance between the rear base point and the wake stagnation point, and the separation angle $\theta_{s}$, as indicated in figure 2 .

The non-dimensional pressure and stresses are expressed as the pressure coefficient, $c_{p}$, the skin friction coefficient, $c_{f}$, and the normal stress coefficient, $c_{n}$, respectively, by

$$
c_{p}=\frac{p-p_{\infty}}{\rho_{\infty} u_{\infty}^{2} / 2}, \quad c_{f}=\frac{\sigma_{r \theta}}{\rho_{\infty} u_{\infty}^{2} / 2} \quad \text { and } \quad c_{n}=\frac{\sigma_{r r}}{\rho_{\infty} u_{\infty}^{2} / 2},
$$

in which $\sigma_{r \theta}$ and $\sigma_{r r}$ are the shear and normal stress components, respectively, in the cylindrical coordinate system $(r, \theta)$ illustrated in figure 1 . The drag on the circular cylinder is composed of three separate components, namely pressure drag $F^{p}$, skin friction drag $F^{f}$, and normal stress drag $F^{n}$. The corresponding components of the drag coefficient, $C_{D}^{p}, C_{D}^{f}$ and $C_{D}^{n}$, can be estimated from the computational solution, respectively, by

$$
C_{D}^{p}=\frac{F^{p}}{\rho_{\infty} u_{\infty}^{2} D / 2}=-\frac{1}{\rho_{\infty} u_{\infty}^{2}} \int_{0}^{2 \pi}\left(p-p_{\infty}\right) \cos \theta \mathrm{d} \theta,
$$




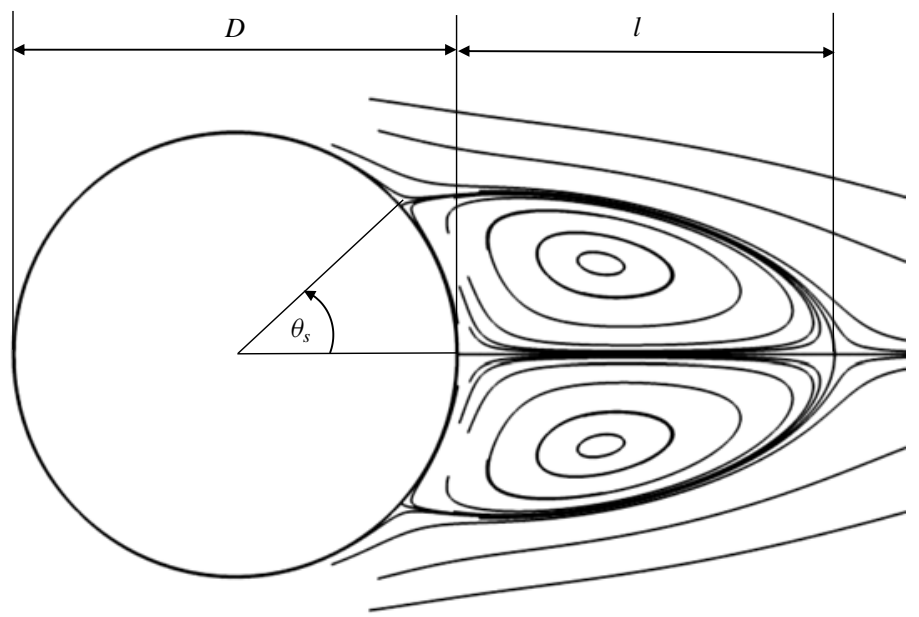

FIGURE 2. Schematics of the circular cylinder and the twin vortices.

$$
C_{D}^{f}=\frac{F^{f}}{\rho_{\infty} u_{\infty}^{2} D / 2}=\frac{1}{\rho_{\infty} u_{\infty}^{2}} \int_{0}^{2 \pi} \sigma_{r \theta} \sin \theta \mathrm{d} \theta
$$

and

$$
C_{D}^{n}=\frac{F^{n}}{\rho_{\infty} u_{\infty}^{2} D / 2}=-\frac{1}{\rho_{\infty} u_{\infty}^{2}} \int_{0}^{2 \pi} \sigma_{r r} \cos \theta \mathrm{d} \theta .
$$

The total drag coefficient, $C_{D}$, is the sum of each component, i.e.

$$
C_{D}=C_{D}^{p}+C_{D}^{f}+C_{D}^{n}
$$

For the convenience of discussion in the following subsections, the drag coefficient in the incompressible continuum limit is denoted as $C_{D, c o n t}$.

\subsection{Drag coefficient in the continuum limit}

In the continuum limit, there are well-documented experimental, theoretical and numerical studies of the drag coefficient, the onset of flow separation, and the geometry of the wake. They are used in the present study to verify the accuracy of the numerical implementation and serve as the baseline to reflect rarefaction and compressibility effects on the flow characteristics as the state of the gas departs from the continuum limit. Incompressible flows in the continuum limit are computed by the Navier-Stokes equations with no-slip boundary conditions for $0.1 \leqslant R e \leqslant 45$. When a fluid flows past a stationary cylinder, the flow is disturbed not only downstream, but also upstream and sideways. An appropriate computational domain size is required to minimise any boundary effects. The ratio of the domain length, $L$, to the width, $H$, is fixed at 10 , while the aspect ratio of $H / D$ varies for different Reynolds numbers. Shown in figure $3(a)$ is the drag coefficient calculated from computational solutions for $R e=0.1,0.2,0.5$ and 1.0, respectively, for different sizes of computational domain. At $R e=0.1$, the aspect ratio, $H / D$, must be greater than 2000 to obtain a grid-independent value of $C_{D \text {,cont }}$, whereas at $R e=1$, the value of $C_{D \text {,cont }}$ requires the computational domain to be $H / D=200$. To reach a converged value of the drag 

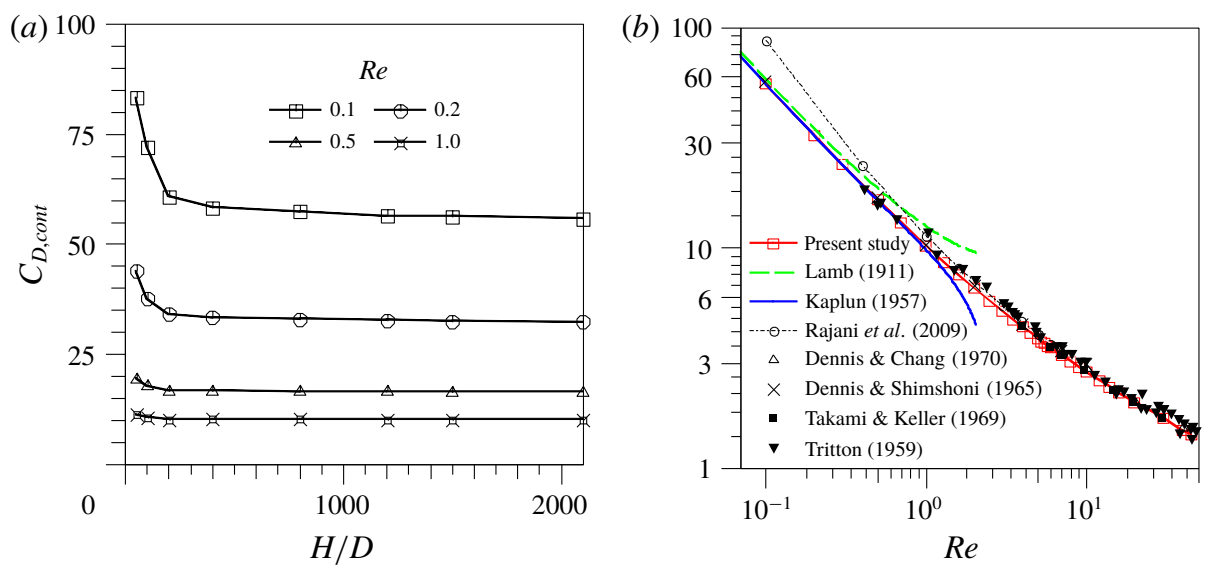

FIGURE 3. (Colour online) (a) Effect of the domain size on the computed values of the drag coefficient at different Reynolds numbers. (b) Comparison of the drag coefficient at different Reynolds number for incompressible continuum flow by different approaches.

coefficient, $C_{D, c o n t}$, a large computational domain is required for low-Reynolds-number flow. In the present study, all computations have been obtained using a sufficiently large domain for the specific Reynolds number under investigation.

The computed values of $C_{D, c o n t}$ for incompressible continuum flows are plotted against the Reynolds number in figure 3(b), in comparison with theoretical and experimental data. In the creeping flow regime, $R e<1$, our computed value for $C_{D, c o n t}$ is slightly lower than the solution of Lamb (1911), which is

$$
C_{D, \text { cont }}=\frac{8 \pi}{\operatorname{Re}} \varepsilon
$$

in which

$$
\varepsilon=\left[\frac{1}{2}-\gamma_{e}-\ln \left(\frac{R e}{8}\right)\right]^{-1},
$$

where $\gamma_{e}=0.577215$ is Euler's constant. The present results are in good agreement with the asymptotic solution of Kaplun (1957):

$$
C_{D, c o n t}=\frac{8 \pi}{R e} \varepsilon\left(1-0.87 \varepsilon^{2}\right),
$$

which is a higher-order approximation than Lamb's solution. Skinner (1975) achieved an even higher-order expansion than Kaplun's approximation, but the difference between them is negligible. The computational solutions of Rajani, Kandasamy \& Majumdar (2009) overpredict the value of $C_{D, c o n t}$ significantly, particularly at small values of Reynolds number, as indicated in figure $3(b)$, as their study used a small computational domain. In the region $R e>1$, our computed values of $C_{D, c o n t}$ agree well with the experimental data (Tritton 1959) and the computational results of Dennis \& Shimshoni (1965), Takami \& Keller (1969) and Dennis \& Chang (1970). 


\subsection{Drag coefficient in the slip and early transition regime}

When the state of the gas is no longer in equilibrium, the characteristics of the flow are determined not only by the Reynolds number, but also by the Knudsen number. Yamamoto \& Sera (1985) obtained an approximation of the drag coefficient for $R e<1$ based on the linearised BGK equation. Following the notation used in the present paper, their drag coefficient can be written as

$$
C_{D}=\frac{8 \pi}{R e} \xi
$$

where

$$
\xi=\left[\frac{1}{2}-\gamma_{e}-\ln \left(\frac{R e}{8}\right)+\frac{4 K n_{\infty}}{\sqrt{\pi}} \Lambda\right]^{-1},
$$

and $\Lambda$ is a parameter close to unity in the slip and early transition regime and gradually increases to 1.5 towards the free-molecular regime. The values of $\Lambda$ are given in table I of Yamamoto \& Sera (1985). As $K n_{\infty} \rightarrow 0$, equations (5.9) and (5.10) reduce to Lamb's solution. As a close analogy to Kaplun's higher-order correction to Lamb's solution, an empirical modification to (5.9) is proposed in the present study as

$$
C_{D}=\frac{8 \pi}{\operatorname{Re}} \xi\left(1-0.87 \xi^{2}\right),
$$

so that, as $K n_{\infty} \rightarrow 0$, equation (5.11) reduces to Kaplun's solution in the continuum limit. Figure 4(a) shows the predictions from Yamamoto \& Sera's approximation (5.9) and its modification (5.11) at $R e=0.5$. The gap between the two predictions is the difference between Lamb and Kaplun's solutions as $K n_{\infty} \rightarrow 0$. However, when $K n_{\infty}>1$, the difference between (5.9) and (5.11) diminishes. With the combination of the concept of a 'molecular layer' around the cylinder and Oseen's approximation by Lamb away from the cylinder, Pich (1969) obtained an expression for $C_{D}$ in the transition regime:

$$
C_{D}=\frac{8 \pi}{R e}\left[\frac{1}{2}-\gamma_{e}-\ln \left(\frac{R e}{8}\right)+1.747 K n_{\infty}-\ln \left(1+0.749 K n_{\infty}\right)\right]^{-1} .
$$

Naturally, when $K n_{\infty} \rightarrow 0$, equation (5.12) recovers Lamb's solution and is close to the solution of Yamamoto \& Sera (1985) in the slip and transition regime, as shown in figure $4(a)$.

Figure $4(b)$ presents the values of $C_{D}$ calculated for $R e=0.5$ from the numerical solution of the NSF equations with velocity-slip and temperature-jump boundary conditions, the R13 and R26 equations in the slip and early transition regime along with (5.11) and the DSMC data. The numerical solutions from all three macroscopic models converge to Kaplun's theoretical value as $K n_{\infty} \rightarrow 0$. As expected, the value of $C_{D}$ decreases as $K n_{\infty}$ increases at a constant $R e$. When $K n_{\infty}<0.07$, all three numerical solutions are in close agreement with the modified Yamamoto \& Sera approximation, equation (5.11). As $K n_{\infty}$ increases above 0.07 , the NSF equations with slip boundary conditions start to deviate from the other solutions and overpredict the value of $C_{D}$ significantly beyond the slip regime. The R13 equations follow the R26 results and (5.11) up to $K n_{\infty}=0.3$. Above this value, the R13 model underpredicts the value of $C_{D}$ while the R26 equations are in good agreement with (5.11) up to a Knudsen number of approximately unity. This is due to the fact that the R26 model 

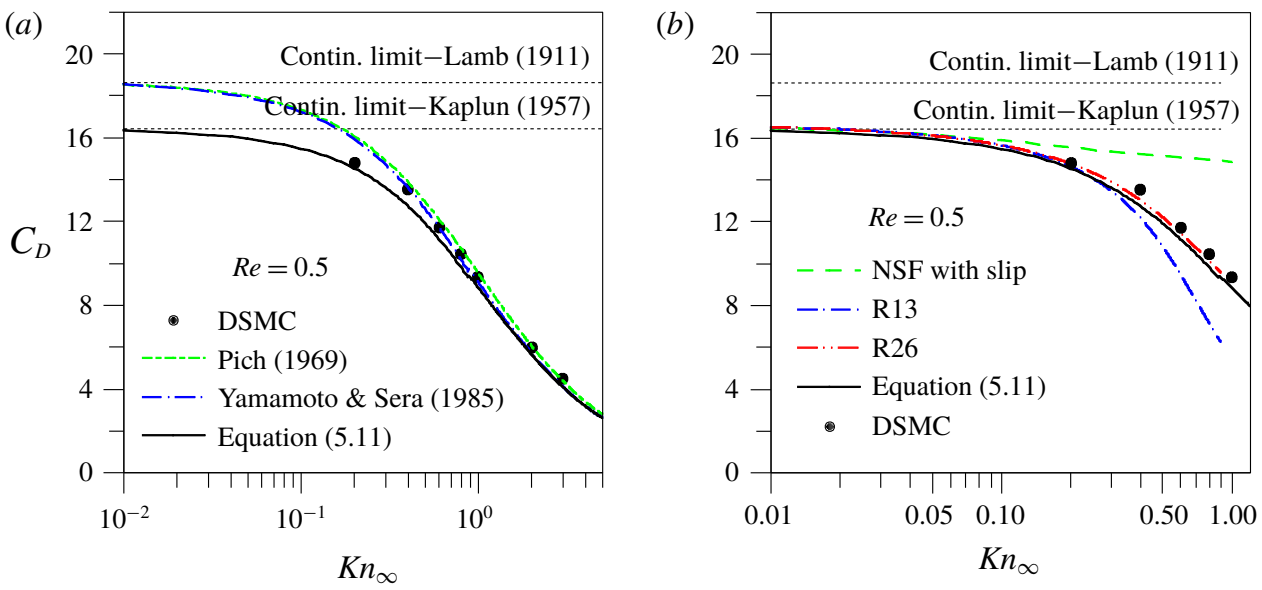

FIGURE 4. (Colour online) (a) Predicted drag coefficient from the BGK kinetic equations. (b) Predicted values of $C_{D}$ from macroscopic models against $K n_{\infty}$ in comparison with DSMC data at $R e=0.5$.

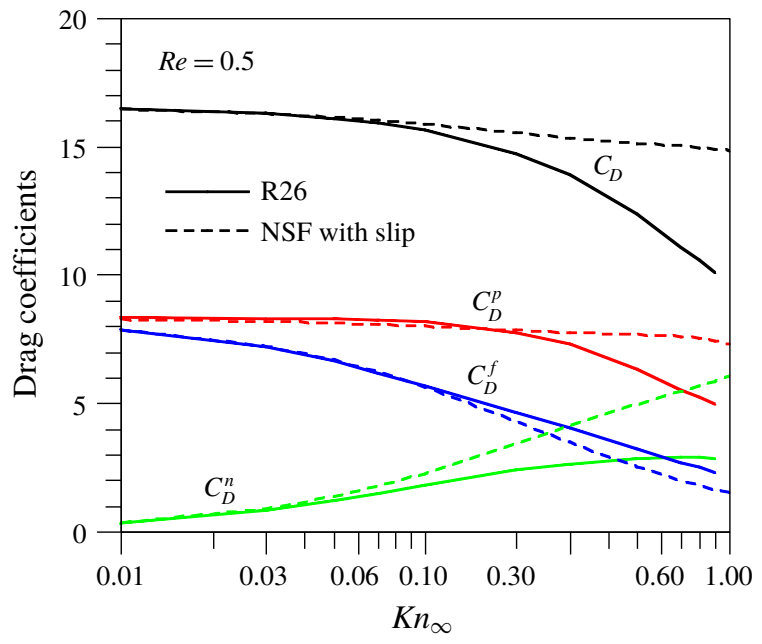

FIgURE 5. (Colour online) Predicted components of $C_{D}$ from macroscopic models at $R e=0.5$ against $K n_{\infty}$.

is able to capture the Knudsen layer much more accurately than the R13 model (Gu, Emerson \& Tang 2009; Gu et al. 2010; Young 2011; Gu \& Emerson 2014). It is computationally expensive for DSMC to simulate flows with such low Reynolds and Knudsen numbers. Consequently, only limited simulations at moderate Knudsen numbers are shown in figure 4 . The values of $C_{D}$ from the DSMC simulations are close to (5.11) but are slightly higher.

Using the R26 equation solutions, we can now examine the effect of the Knudsen number on the contribution from the pressure and stress terms (tangential and normal) of the drag coefficient, $C_{D}$. At $K n_{\infty}=0.01$, as shown in figure 5 for $\operatorname{Re}=0.5,50 \%$ of the drag coefficient is generated by the pressure component, $C_{D}^{p}$, while $48 \%$ of 

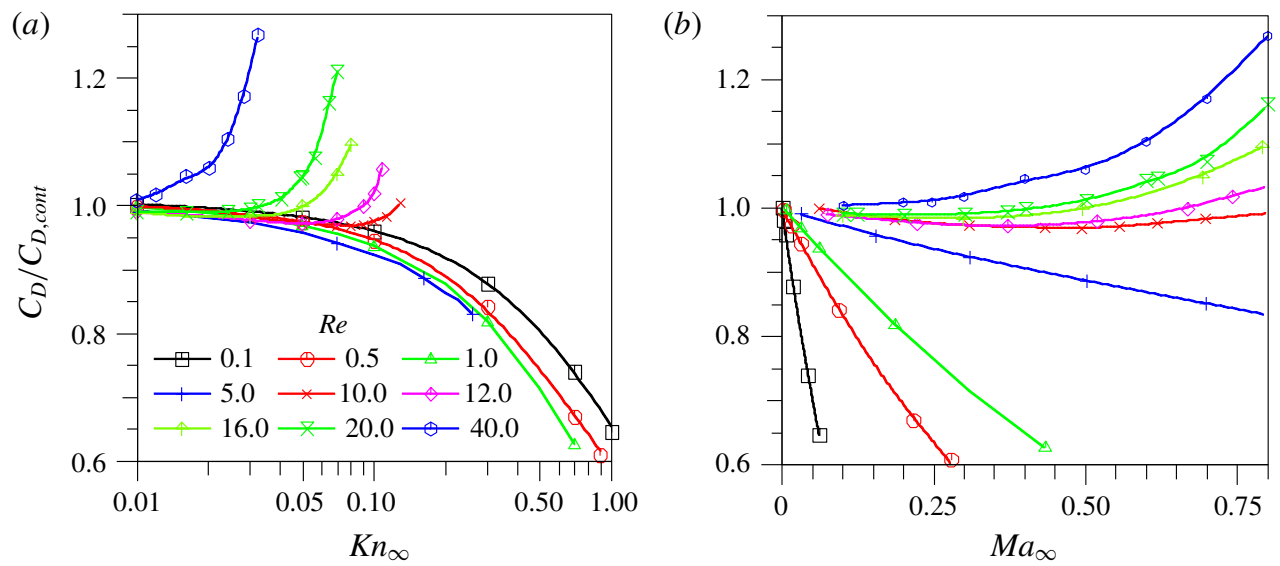

Figure 6. (Colour online) Predicted values of the drag coefficient by the R26 equations for different Reynolds numbers plotted against $(a) K n_{\infty}$ and (b) $M a_{\infty}$.

the value of $C_{D}$ is from the tangential shear stress component, $C_{D}^{f}$. The normal stress component, $C_{D}^{n}$, only contributes $2 \%$ of drag. Indeed, at the continuum limit, there is no contribution from the normal stress towards $C_{D}$. As $K n_{\infty}$ increases, the value of $C_{D}^{n}$ increases and the friction drag decreases, respectively. The value of $C_{D}^{p}$ remains constant until just beyond the slip regime $\left(K n_{\infty} \sim 0.2\right)$ and it then reduces as $K n_{\infty}$ continues to increase. At $K n_{\infty} \approx 0.9$, the proportions of $C_{D}^{p}, C_{D}^{n}$ and $C_{D}^{f}$ contributing to $C_{D}$ are $49.5 \%, 28 \%$ and $22.5 \%$, respectively. Although both $C_{D}^{p}$ and $C_{D}$ reduce as $K n_{\infty}$ increases beyond the slip flow regime, their ratio changes due to the increasing contribution of the normal stress component, which begins to asymptote at $K n \sim 1$. The contribution of $C_{D}^{f}$ decreases as $K n_{\infty}$ increases. The solution of the NSF equations with the velocity-slip boundary condition (represented by the dashed lines in figure 5) follows that of the R26 equations (represented by the solid lines) up to $K n_{\infty} \sim 0.07$. Beyond this value, the NSF equations overpredict $C_{D}^{n}$ and $C_{D}^{p}$ in comparison with the R26 equations, which results in an overprediction of the total drag.

The non-equilibrium effects on the value of the drag coefficient for the range of Reynolds number considered are quite distinct. For small Reynolds numbers, $R e<10$, the drag coefficient decreases below its corresponding value at the continuum limit as $K n_{\infty}$ increases, as shown in figure $6(a)$. When $R e \geqslant 10$, the ratio of rarefied to continuum drag, $C_{D} / C_{D, c o n t}$, shows a slight initial drop close to the continuum regime. However, as $K n_{\infty}$ increases, the drag ratio is seen to increase above unity. The larger the Reynolds number, the more obvious the trend is. As indicated by (4.5), both rarefaction and compressibility are related. Although rarefaction increases the velocity slip around the cylinder and tends to reduce the drag coefficient, the effects of compressibility increase the pressure drop across the cylinder and increase the drag coefficient. This will be discussed further in $\S 5.4$. The values of $C_{D} / C_{D, \text { cont }}$ plotted against $M a_{\infty}$ are presented in figure $6(b)$ for the same range of Reynolds number as in figure 6(a). For flows with $R e>10$, the drag coefficient will be above its corresponding continuum value as the Mach number increases. Although it is difficult to separate the effects of rarefaction and compressibility, figure 6 implies that compressible effects begin to dominate when $R e>10$, whereas rarefied gas effects are stronger when $R e<10$ (Hu et al. 2009). 

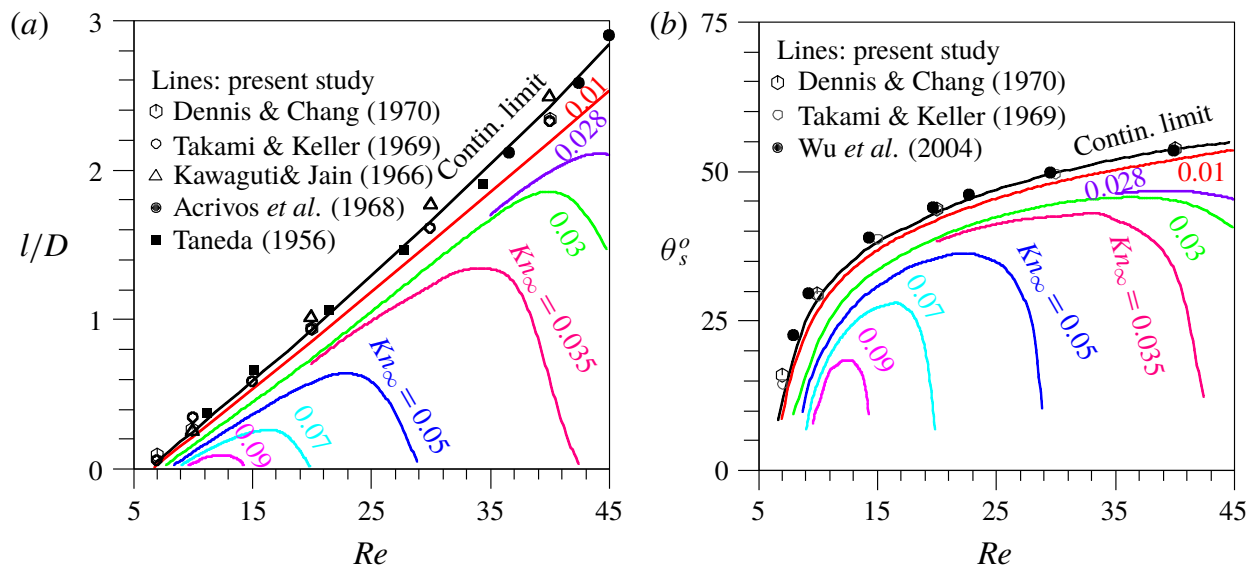

FIgURE 7. (Colour online) Predicted vortex size from the R26 equations against $R e$ for a range of $K n_{\infty}$ in comparison with the continuum limit: $(a)$ wake length and $(b)$ separation angle.

\subsection{Non-equilibrium effects on vortex formation and size}

In the continuum limit, a pair of steady twin vortices is formed downstream of the cylinder when the Reynolds number, $R e$, is above a critical value, denoted by $R e_{\text {onset }}$. The onset of flow separation can be detected by gradually increasing the Reynolds number. Above a critical value, $R e_{\text {onset }}$, the streamlines of the flow start to detach from the cylinder and a pair of symmetric vortices begin to form. The length of the twin vortices behind the cylinder, denoted by $l$, and the separation angle, $\theta_{s}$, as illustrated in figure 2, are measured against the Reynolds number. The critical Reynolds number can be found by extrapolating $l$ to zero. Our simulations predict a value of 6.5 , which is close to the value of 6.2 obtained by Dennis \& Chang (1970). The size of these vortices grows as $R e$ increases until the Reynolds number reaches a second critical value, $R e_{c}$; beyond this value, the vortices become unstable. Kumar \& Mittal (2006) reviewed the values of the second critical Reynolds number obtained from numerical simulations and experimental observations, and found that it varied from 40 to 50. In their work, they obtained a value of $R e_{c}$ just above 47 by both direct time integration and linear stability analysis methods. However, as the focus of the present study is on steady-state flows, we only consider Reynolds numbers below this critical value, $R e_{c}$.

The relationship between the Reynolds number and the normalised vortex length, $l / D$, has been studied, experimentally and numerically, for continuum incompressible flows by many researchers. Experimental data measured by Taneda (1956) and Acrivos et al. (1968) for liquid flow past a circular cylinder are indicated by the solid symbols in figure 7 $(a)$ along with data from numerical solutions of the incompressible NavierStokes equations by Kawaguti \& Jain (1966), Takami \& Keller (1969) and Dennis \& Chang (1970), indicated by the hollow symbols. They are all in good agreement with the values of $l / D$ from the present numerical solution of the incompressible Navier-Stokes equations represented by the solid line for the continuum limit. The present values of the corresponding separation angle, $\theta_{s}$, plotted in figure $7(b)$ by the uppermost solid line, are slightly lower than the experimental measurements of $\mathrm{Wu}$ et al. (2004) when $R e$ is small but agree well with the computational results of Takami \& Keller (1969) and Dennis \& Chang (1970). 
(a)

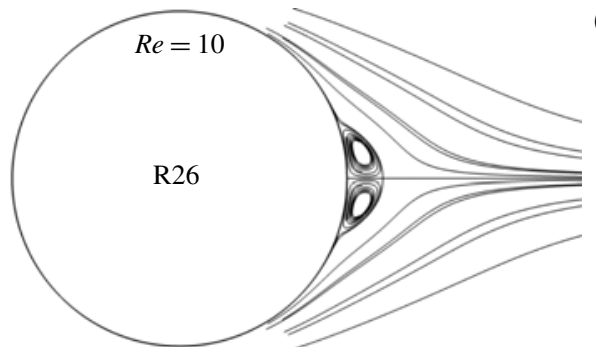

(b)

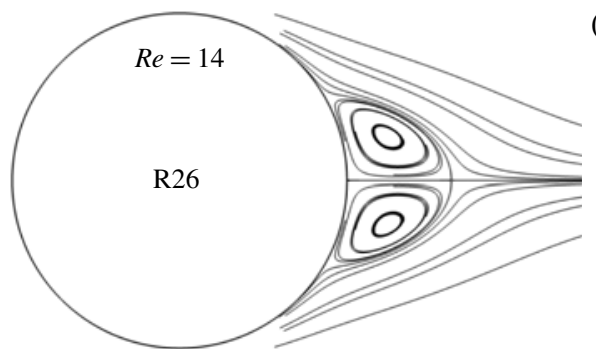

(c)

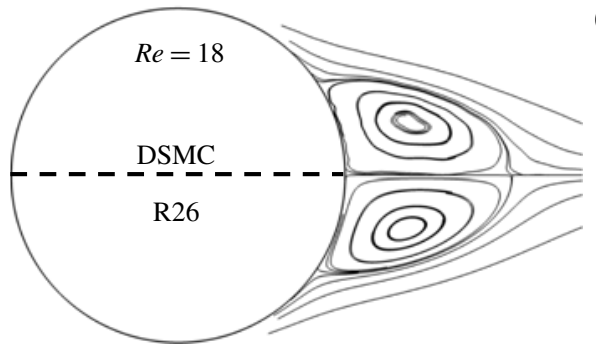

(d)

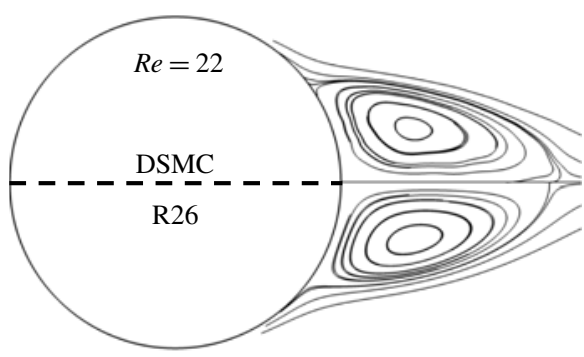

$(e)$

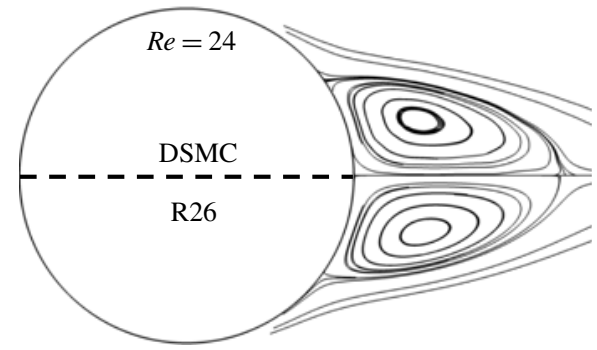

$(f)$

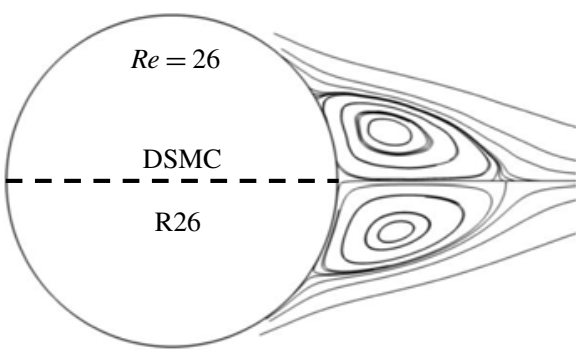

$(g)$

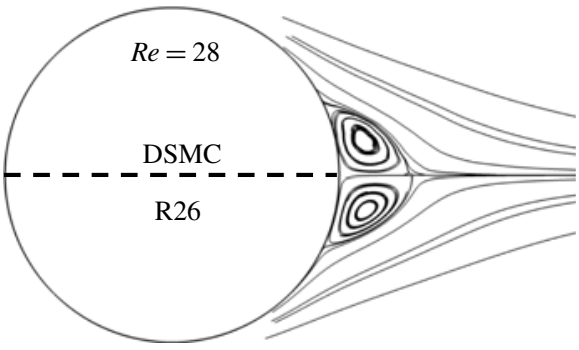

(h)

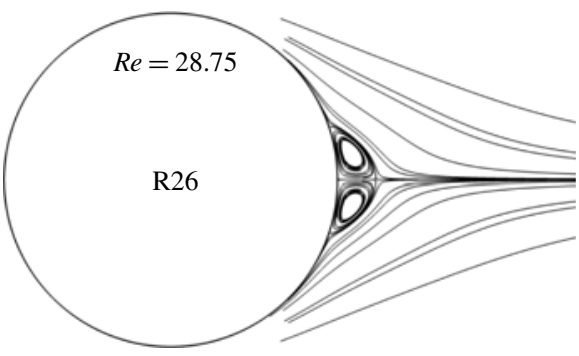

FIGURE 8. The streamlines behind the cylinder calculated from the R26 equation solution and the DSMC data at $K n_{\infty}=0.05$ for eight different Reynolds numbers.

The lines second from the top in figure $7(a, b)$ are the values of $l / D$ and $\theta_{s}$ predicted by the R26 moment equations for gas flow at $K n_{\infty}=0.01$. The vortex length is slightly reduced and the separation point moves in the downstream direction, in comparison to continuum flow, due to non-equilibrium effects at the wall, particularly at larger Reynolds numbers. Within the computed range of Reynolds number, $R e \leqslant 45$, the values of $l / D$ and $\theta_{s}$ increase as $R e$ increases for this particular Knudsen number. However, when $K n_{\infty}>0.028$, as shown in figure 7, an interesting phenomenon occurs, which has not been observed in continuum flow. Figure 8 shows the streamlines behind the cylinder calculated from the computational results of the R26 equations and the DSMC data at $K n_{\infty}=0.05$ as the Reynolds number increases from 10 to 

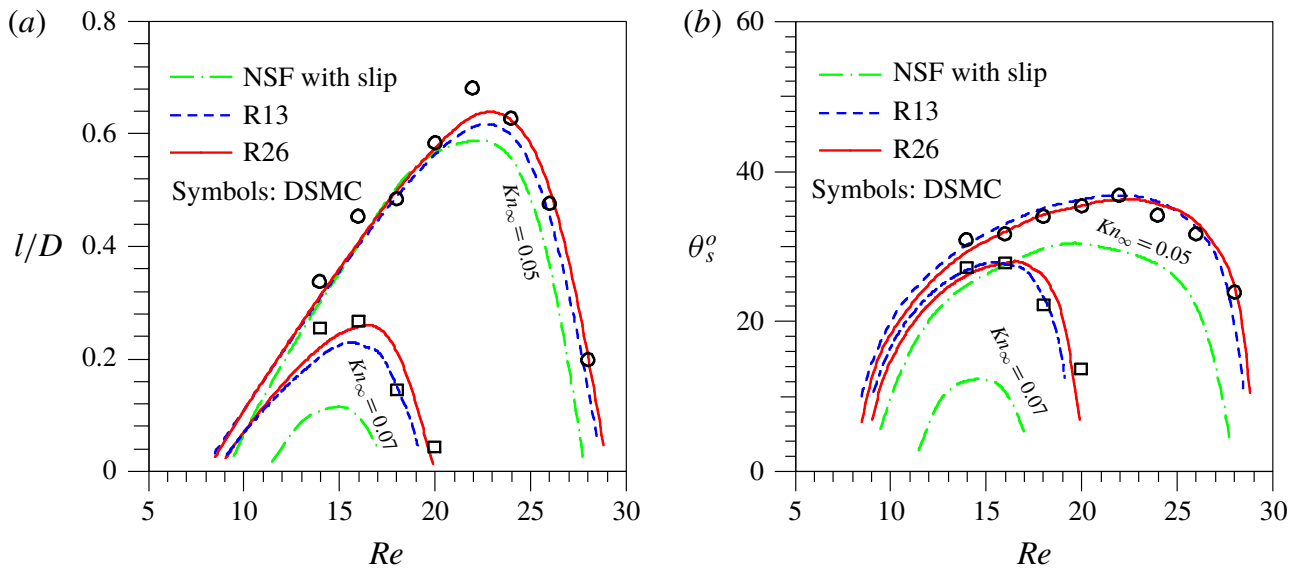

FIGURE 9. (Colour online) Predicted vortex size from the macroscopic models in comparison with the DSMC data: $(a)$ wake length and $(b)$ separation angle. Lines: R26. Symbols: DSMC, $K n_{\infty}=0.05(\bigcirc)$ and $K n_{\infty}=0.07$ ( $\left.\square\right)$.

28.75. The vortex length initially increases as $R e$ increases and the separation point moves upstream, as illustrated by $(a-d)$ in figure 8 . However, when the Reynolds number is above a critical value, $R e_{c l}$, the twin vortices gradually diminish in size as $R e$ increases until they eventually disappear, as demonstrated by figure $8(e-g)$. The agreement between the R26 equations and the DSMC data is excellent, apart from the stagnation point region where the gas velocity is close to zero and the statistical noise in the DSMC data is high.

All three macroscopic models, the NSF, R13 and R26 equations, can capture the general phenomenon but differ considerably in capturing the physical detail, as demonstrated in figure 9. In the vortex-growing region, for $K n_{\infty}=0.05$, the vortex lengths predicted by the three models lie close to each other before they reach their respective maximum length. The R26 and NSF equations predict the longest and shortest maximum length, respectively, with the R13 equations close to the R26. The vortex length predicted by DSMC for $K n_{\infty}=0.05$, shown in figure $9(a)$ by the open symbols, is generally closer to the R26 model than the other two macroscopic models. However, the separation point predicted by the NSF equations with the velocity-slip boundary condition is significantly different from the moment equations and the DSMC data, as illustrated in figure $9(b)$. At $K n_{\infty}=0.07$, within the traditional slip regime, the discrepancy between the NSF and moment equations is quite substantial. The NSF equations underpredict the vortex size significantly, in terms of both wake length and separation angle.

The Reynolds numbers relating to the onset and end of the symmetric vortices behind the cylinder, $R e_{\text {onset }}$ and $R e_{\text {end }}$, can be obtained by extrapolating the curves in figures 7 and 9 to $l / D=0$ and are plotted against Knudsen number in figure $10(a)$ for the three macroscopic models. The values of $R e_{\text {onset }}$ obtained from both the R13 and R26 equations are almost identical and increase gradually as $K n_{\infty}$ increases. Conversely, the values of $R e_{\text {end }}$ from the R13 are close to but slightly lower than those from the R26 equations. From figure 10, we see that the NSF equations predict a much narrower region where the twin vortices can exist in comparison to the moment equations, as indicated by the broken lines in figure $10(a)$, despite the fact that this is clearly in the traditional slip regime. This significant discrepancy shows 

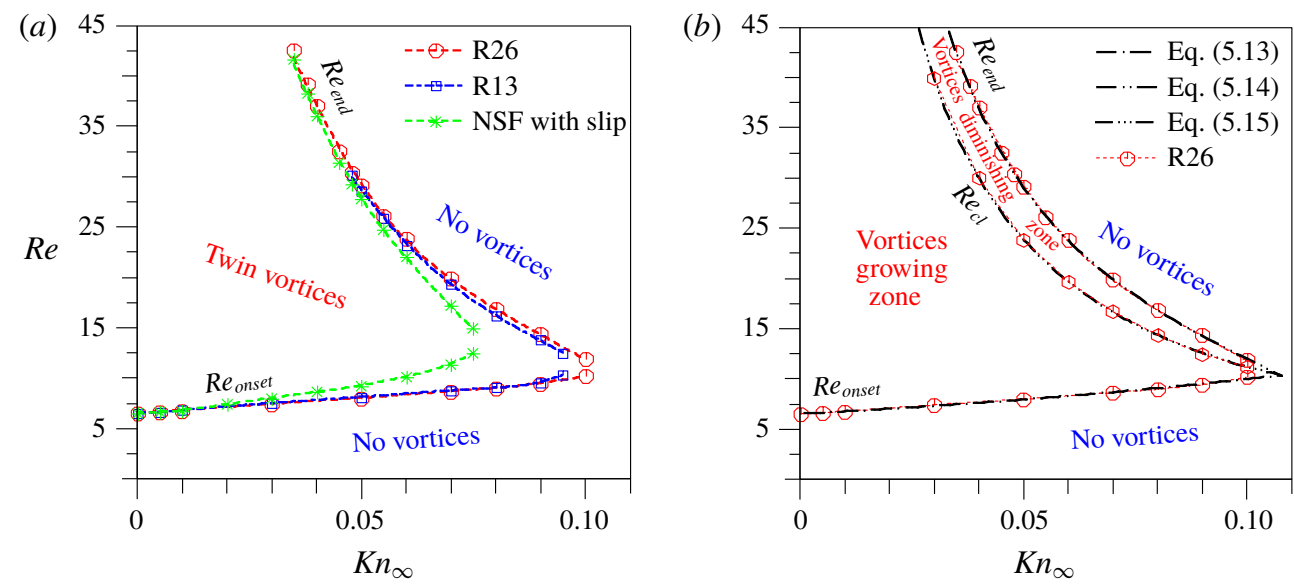

FIGURE 10. (Colour online) Vortex existence $R e-K n_{\infty}$ diagram of non-equilibrium gas behind a circular cylinder.

that care is needed when using the NSF equations with velocity slip, even when operating in the traditional slip flow regime. Plotted in figure $10(b)$ is the critical Reynolds number, $R e_{c l}$, which divides the twin vortex region into a zone in which the vortices are growing and one where the vortices are diminishing.

Empirical expressions for $R e_{\text {onset }}, R e_{\text {end }}$ and $R e_{c l}$ are obtained, respectively, by curve fitting the data from the R26 equations, as

$$
\begin{gathered}
R e_{\text {onset }}=6.5+2.169 \times\left(10 K n_{\infty}\right)+1.275 \times\left(10 K n_{\infty}\right)^{2}, \\
R e_{\text {end }}=\frac{1}{K n_{\infty}} \sqrt{\frac{\gamma \pi}{2}}-5.886+8.862 \times\left(10 K n_{\infty}\right)-7.190 \times\left(10 K n_{\infty}\right)^{2}
\end{gathered}
$$

and

$$
R e_{c l}=\frac{0.685}{K n_{\infty}} \sqrt{\frac{\gamma \pi}{2}}+5.598-9.927 \times\left(10 K n_{\infty}\right)+4.435 \times\left(10 K n_{\infty}\right)^{2} .
$$

The steady twin vortices occur behind the cylinder only in the range $R e_{\text {onset }}<R e<$ $R e_{e n d}$. By extrapolating equations (5.13) and (5.14) in figure 10(b), they meet roughly at $K n_{\infty}=0.108$. In other words, no vortices will exist downstream of the cylinder, for any Reynolds number, when $K n_{\infty}>0.108$.

\subsection{Flow physics around the cylinder}

One of the main non-equilibrium phenomena associated with a rarefied gas is the velocity slip at the solid surface. The slip velocity, $u_{\tau}$, is determined by the wall shear stress, tangential heat flux along the surface, and other higher-order moments, as expressed through the boundary condition, equation (2.13). Since the flow configuration under consideration is symmetric and the polar coordinates $(r, \theta)$ are anticlockwise, it is intuitive to plot $-u_{\tau} / u_{\infty}$ around the cylinder from the front stagnation point, at $\theta=180^{\circ}$, to the cylinder base, at $\theta=0^{\circ}$. Figure 11 shows the velocity slip for three different Reynolds numbers and various Knudsen numbers from solutions obtained from the R26 equations and DSMC. For fixed values of $R e$ and 


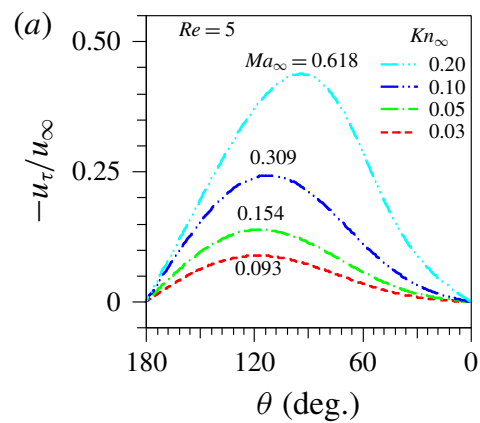

(b)

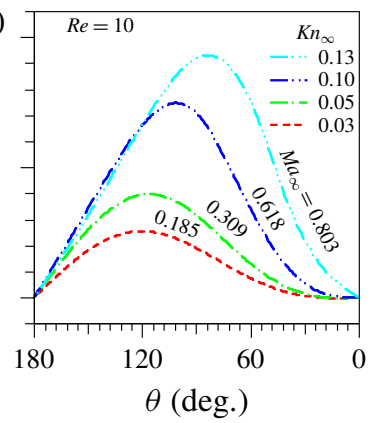

(c)

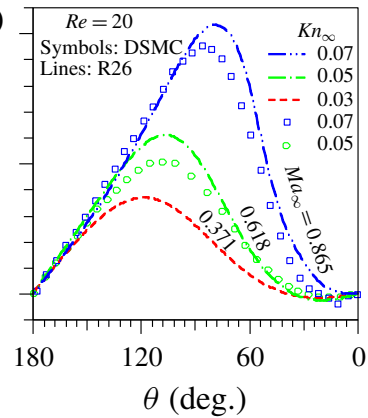

FIGURE 11. (Colour online) Velocity slip around the cylinder for different $R e$ and $K n_{\infty}$. Lines: R26. Symbols: DSMC, $K n_{\infty}=0.05$ (○) and $K n_{\infty}=0.07$ ( $\square$ ).
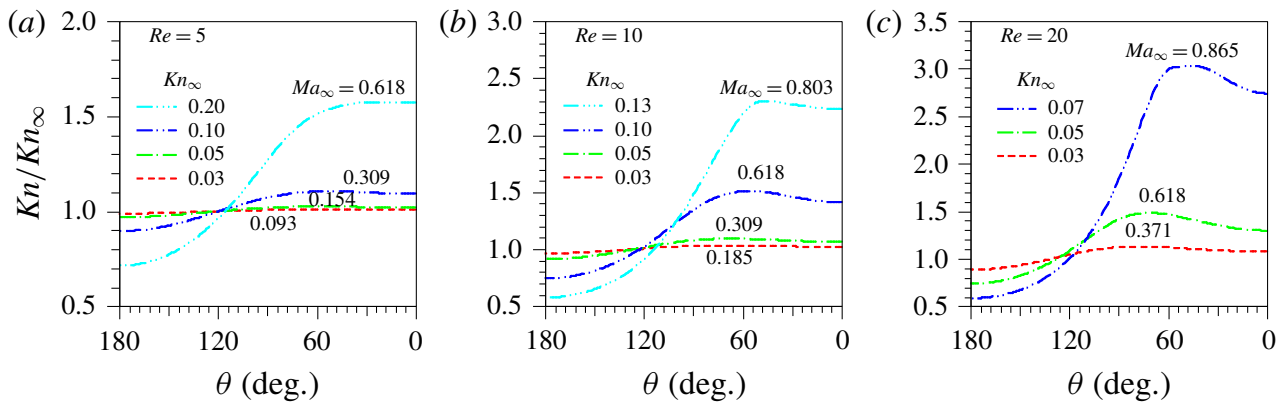

FIgURE 12. (Colour online) The local Knudsen number around the cylinder.

$K n_{\infty}$, the velocity slip along the cylinder increases from the front stagnation point and reaches a maximum value before it drops to zero at $\theta=0^{\circ}$. The location of the maximum slip depends on the value of $R e$ and $K n_{\infty}$. For example, when $R e=5$ and $K n_{\infty}=0.03$, the maximum velocity slip occurs at $\theta=120^{\circ}$ in figure $10(a)$, while it occurs at $\theta=80^{\circ}$ for $R e=20$ and $K n_{\infty}=0.07$, as shown in figure $11(c)$. As anticipated, the velocity slip increases with $K n_{\infty}$ for a fixed value of $R e$. Comparing figure 11(a) and $(c)$, the velocity slip clearly increases with Reynolds number for a fixed value of $K n_{\infty}$. However, this does not imply that it is purely a Reynolds-number effect. In comparison to the DSMC data in figure $11(c)$, the R26 equations provide a reliable prediction of the velocity slip around the front half of the cylinder but tend to overpredict the maximum slip velocity in the leeward region.

Equation (4.5) indicates that $M a_{\infty}$ increases with the product of $R e$ and $K n_{\infty}$. As the values of $M a_{\infty}$, which are illustrated in figure 11, are above 0.3 , there will be compressibility effects in the solution. Consequently, the local non-equilibrium state of the gas will differ from its undisturbed free-stream value. The local Knudsen number, $K n$, determined from the local temperature and pressure, is no longer the same as $K n_{\infty}$. In figure 12 , we have plotted the ratio, $K n / K n_{\infty}$, for three Reynolds numbers and different values of $K n_{\infty}$ or $M a_{\infty}$ around the cylinder. When $M a_{\infty}<0.3$, the value of $K n / K n_{\infty}$ around the cylinder is approximately equal to unity, i.e. the local nonequilibrium state is the same as the nominal one defined by the free stream. The value of $K n / K n_{\infty}$ is less than unity at the front of the cylinder when $M a_{\infty}>0.3$, and when $\theta<120^{\circ}$ it increases beyond one, as shown in figure 12(a). The larger the value of $M a_{\infty}$, the more obvious the phenomenon, as indicated in figure 12. In the case of 

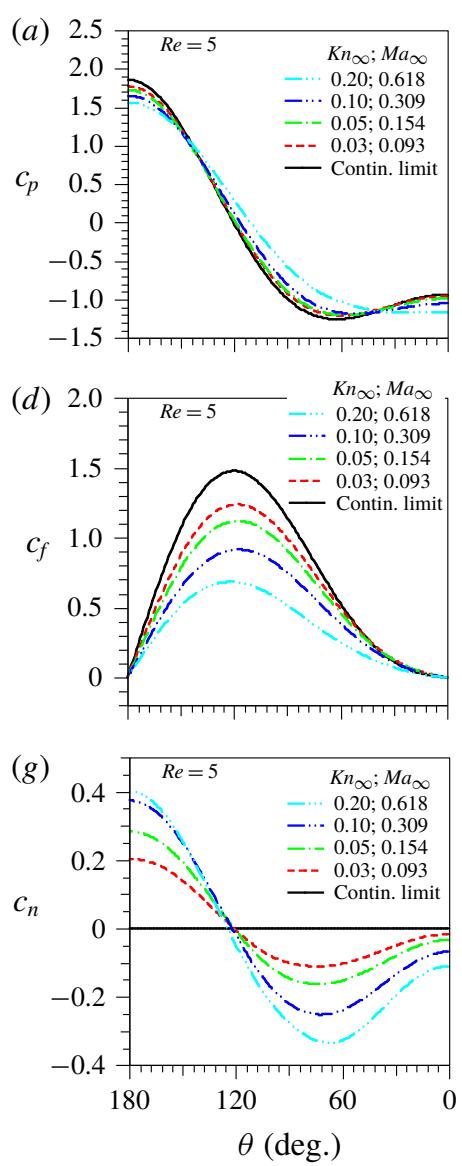

(b)

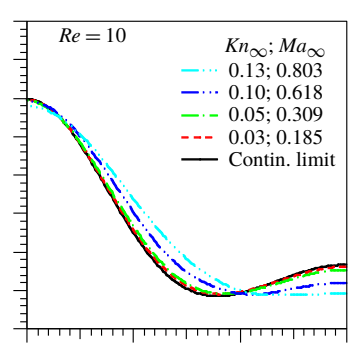

(e)

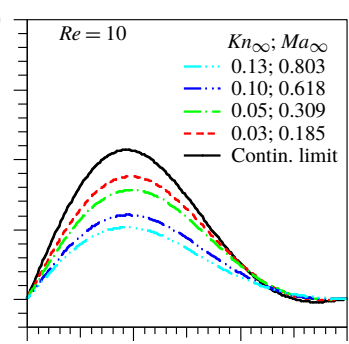

(h)

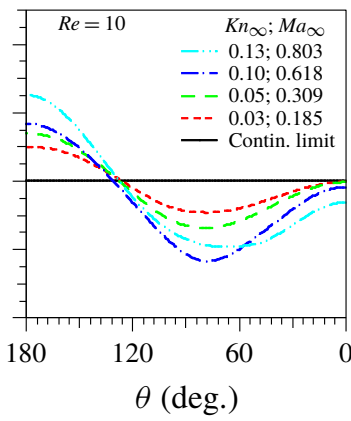

(c)

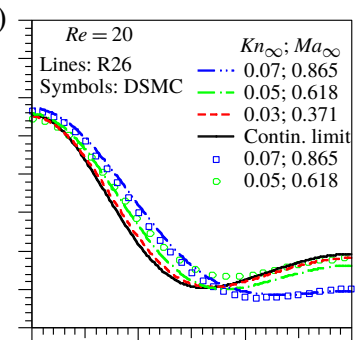

$(f)$

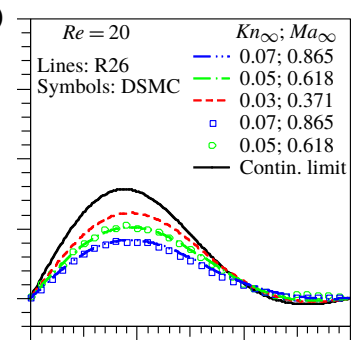

(i)

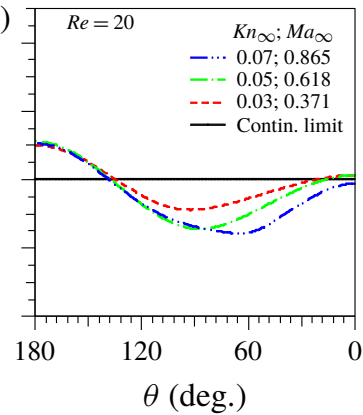

FIgURE 13. (Colour online) Viscous and rarefaction effects on $(a-c) c_{p},(d-f) c_{f}$ and $(g-i) c_{n}$ around the cylinder wall. Lines: R26. Symbols: DSMC, $K n_{\infty}=0.05$ (O) and $K n_{\infty}=0.07(\square)$.

$R e=20$ and $K n_{\infty}=0.07$, the local Knudsen number, $K n$, is only half of its nominal value at the front, while figure $12(c)$ shows that it exceeds $3 K n_{\infty}$ at the rear of the cylinder. Although it is in the slip regime in terms of the free-stream Knudsen number, $K n_{\infty}$, the flow around the cylinder experiences a significant shift from the slip regime well into the transition regime. The high value of velocity slip around the rear section of the cylinder moves the separation point further downstream with an increase of $R e$ and is able to overcome the adverse pressure gradient to reduce the vortex size until it eventually disappears, as shown in figures 8 and 9. The strong variation in local Knudsen number could also explain the discrepancy between DSMC and the R26 equations in predicting velocity slip, as highlighted in figure 11(c).

The high value of local Knudsen number results in the pressure coefficient, $c_{p}$, the skin friction coefficient, $c_{f}$, and the normal stress coefficient, $c_{n}$, deviating significantly from their continuum limits. Figure 13 illustrates the variation in surface value of the coefficients around the cylinder for three Reynolds numbers and various Knudsen numbers. At $R e=5$, the value of $c_{p}$ is below its continuum limit at the front and rear of the cylinder for all $K n_{\infty}$ or $M a_{\infty}$ considered. It then rises above the continuum limit for $145^{\circ}>\theta>45^{\circ}$, approximately, as shown in figure $13(a)$. At $R e=10$, the value 

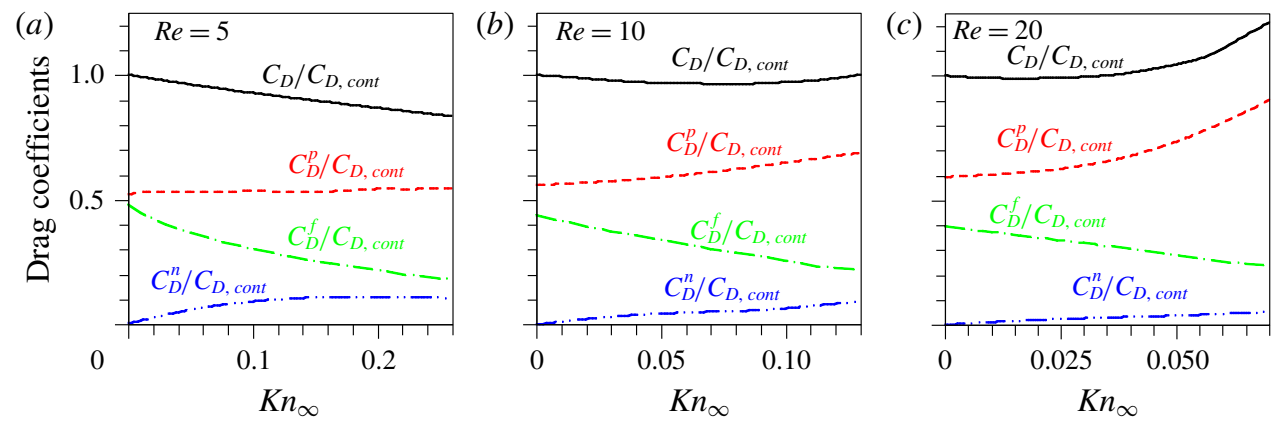

FIGURE 14. (Colour online) Viscous and rarefaction effects on predicted components of $C_{D}$ from the R26 equations.

of $c_{p}$ lies close to its continuum value at the front stagnation point for $K n_{\infty} \leqslant 0.1$. However, the value of $c_{p}$ clearly rises above the continuum limit until $\theta \simeq 60^{\circ}$. In contrast, at $R e=20$, shown in figure 13(c), the value of $c_{p}$ is generally above the continuum value at $\theta=180^{\circ}$. For all Reynolds numbers considered, the value of $c_{p}$ for the rarefied gas is always lower than the continuum limit at the rear of the cylinder, i.e. $\theta=0^{\circ}$. Moreover, figure $13(a-c)$ clearly illustrates the separation point moving towards the rear of the cylinder. The flow undergoes compression around the front half of the cylinder, so that $K n / K n_{\infty}<1$, while the gas expands around the rear half of the cylinder, where $K n / K n_{\infty}>1$, as indicated in figure $12(c)$. The variation of $c_{p}$ predicted by the R26 equations is validated by the DSMC data, as shown in figure 13(c).

The skin friction coefficient, $c_{f}$, is zero at the front stagnation point and the cylinder base and reaches a maximum value at $\theta \approx 110^{\circ}$ for all the Reynolds numbers considered, as shown in figure $13(d-f)$. The maximum value of $c_{f}$ is clearly shown to decrease as $R e$ increases. It can also be seen that the skin friction decreases as $K n_{\infty}$ increases for a fixed Reynolds number. The slightly negative value of $c_{f}$ at the rear of the cylinder is due to the recirculating flow. Our simulations show that the R26 equations and the DSMC data agree well for $c_{f}$ at both $K n_{\infty}=0.05$ and 0.07 , as illustrated in figure $13(f)$. For completeness, the normal stress coefficient, $c_{n}$, is plotted along the cylinder wall and is zero in the continuum limit. The magnitude of $c_{n}$ is much smaller than that of $c_{p}$ and $c_{f}$, but can be seen to increase as $K n_{\infty}$ increases, particularly at low Reynolds number, as shown in figure $13(\mathrm{~g})$.

Non-equilibrium effects from the contributions of $c_{p}, c_{f}$ and $c_{n}$ towards the total drag coefficient are presented in figure 14 for the range of Reynolds numbers considered. The contribution of $c_{n}$ to $C_{D}$ increases as $K n_{\infty}$ increases, but it is small compared to the contributions from $c_{p}$ and $c_{f}$ and does not affect the general trend of $C_{D}$. The pressure component, $C_{D}^{p}$, can be seen to contribute more than $50 \%$ of the total drag and this rises as $R e$ or $K n_{\infty}$ increases. As the Knudsen number increases, the skin friction drag, $C_{D}^{f}$, always decreases for a given Reynolds number due to the velocity slip. The resultant trend of $C_{D}$ against $K n_{\infty}$ for a fixed Reynolds number depends on the competition between $C_{D}^{p}$ and $C_{D}^{f}$. At $R e=5$, the decrease of $C_{D}^{f}$ is faster than the corresponding increase in $C_{D}^{p}$ with increasing $K n_{\infty}$. As a result, $C_{D}$ is lower than its continuum value, i.e. $C_{D} / C_{D, \text { cont }}<1$, as the gas departs from its equilibrium state, as shown in figure 14(a). When $R e=10$, the rates of change of $C_{D}^{p}+C_{D}^{n}$ and $C_{D}^{f}$ are roughly the same but in opposing directions and the value of $C_{D} / C_{D, c o n t}$ remains consistently around unity. However, as $R e$ increases to 20 , shown 

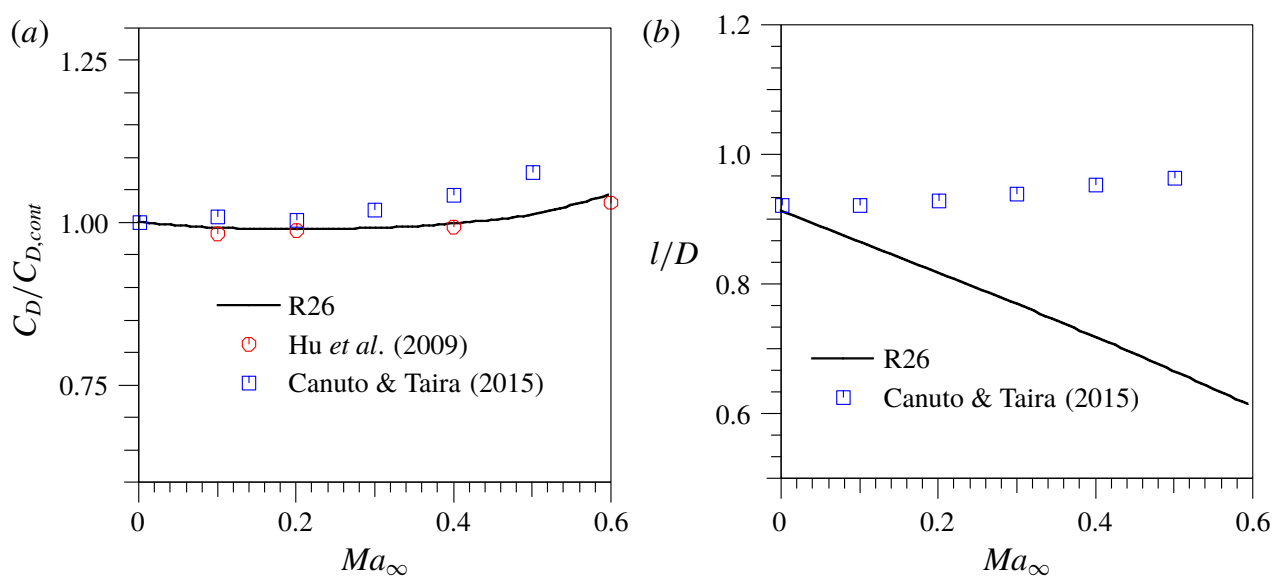

Figure 15. (Colour online) Compressibility effect on $(a) C_{D}$ and $(b) l / D$, with and without non-equilibrium effects at $R e=20$.

in figure $14(c), C_{D}^{p}$ increases much more rapidly with an increase in $K n_{\infty}$ due to the compression and expansion of the gas around the cylinder. As a result, the total drag coefficient, $C_{D}$, rises above its corresponding continuum limit, i.e. $C_{D} / C_{D, \text { cont }}>1$, even though the gas is inside the accepted slip flow limit.

The flow characteristics around the cylinder are determined by a combination of viscous, rarefaction and compressibility effects and the overall behaviour of the flow depends on the complex interplay between these competing effects. Canuto \& Taira (2015) recently studied how compressibility affects the flow without taking into consideration any non-equilibrium effects. They used the compressible NSF equations with the traditional no-slip boundary condition to compute the flow past a cylinder. In their work, the maximum Knudsen number based on the state of the free stream was 0.037 , which occurred for $R e=20$ at $M a_{\infty}=0.5$. By neglecting velocity slip, they overpredict the drag coefficient against $M a_{\infty}$, as shown in figure $15(a)$ for $R e=20$, particularly for $M a_{\infty}>0.3$. Although the Knudsen number for this case is relatively small, it indicates a clear error in the drag prediction and shows that rarefaction effects need to be considered. Hu et al. (2009) studied the same flow conditions with a hybrid strategy of coupling a kinetic approach around the cylinder and a continuum method away from the cylinder. Both rarefaction and compressibility effects were taken into account and their predicted drag coefficient is in good agreement with our results using the R26 equations. The role of the vortex pair is also strongly affected by non-equilibrium effects. Figure 15(b) shows that the vortex length predicted by Canuto \& Taira (2015) increases with $M a_{\infty}$, which is opposite to the prediction by the R26 equations. This illustrates the importance of velocity slip in the determination of the vortex structure.

\section{Conclusions}

The method of moments has been used to investigate rarefaction effects on low-speed compressible flow past a circular cylinder. In particular, we employ the R26 equations to study flow in the slip and early transition regime to understand the impact of non-equilibrium effects on drag. Our results are compared with the Navier-Stokes-Fourier equations and data obtained from the direct simulation Monte 
Carlo method. At the Reynolds numbers considered in this paper, where $0.1 \leqslant R e<45$, solutions are generally obtained under the assumption that the fluid is incompressible with the classic no-slip boundary condition. This approach provides the baseline results for examining how non-equilibrium effects influence the flow physics. For creeping flow, with $R e<1$, a revised expression for the total drag is proposed which approaches Kaplun's rather than Lamb's solution when $K n_{\infty}$ tends to zero. The drag coefficient predicted from the higher moment equations agrees well with kinetic theory when the Knudsen number is less than unity, while the NSF equations consistently overpredict the drag coefficient, even in the slip regime. In the steady flow regime, it has been shown that velocity slip delays flow separation and acts to reduce the size of the vortices downstream of the cylinder. When $K n_{\infty} \geqslant 0.028$, the vortex length increases initially with the increase of $R e$, as observed in the continuum regime. However, once $R e$ is above a critical value, $R e_{c l}$, the lengths of the vortices reduce in size as $R e$ increases until they eventually disappear. An existence criterion for the vortices was shown in a $R e-K n_{\infty}$ diagram. The flow physics around the cylinder was analysed in terms of the velocity slip, pressure and skin friction coefficients. We observed that rarefaction reduces the drag coefficient and the combination of viscous, rarefaction and compressibility effects is intimately linked in determining the flow features, as expressed by the relationship of $R e, K n_{\infty}$ and $M a_{\infty}$. An important factor that has a dramatic effect on the flow is that the local state of the gas around the cylinder, as exemplified by the local Knudsen number, can be significantly different from the free-stream value. It is therefore essential to include all of these effects in the computational studies of subsonic gas flow in the slip and early transition regime even with a moderate or low Reynolds number.

\section{Acknowledgements}

This work was supported by the United Kingdom Engineering and Physical Sciences Research Council (EPSRC) under grants EP/N016602/1 and EP/K038427/1. We extend our gratitude to the Hartree Centre for access to the Blue Wonder computer facility. The DSMC simulations used the UK's National Supercomputing Service, ARCHER; resources were awarded through an ARCHER Resource Allocation Panel award.

Appendix A. Source terms in the moment equations (2.7)-(2.9)

$$
\begin{aligned}
\mathfrak{M}_{i j k}= & -3 \sigma_{\langle i j} \frac{\partial R T}{\partial x_{k\rangle}}+\frac{3 \sigma_{\langle i j}}{\rho}\left(\frac{\partial p}{\partial x_{k\rangle}}+\frac{\partial \sigma_{k\rangle m}}{\partial x_{m}}\right)-\frac{12}{5} q_{\langle i} \frac{\partial u_{j}}{\partial x_{k\rangle}}-3 m_{m\langle i j} \frac{\partial u_{k\rangle}}{\partial x_{m}}, \\
\mathfrak{R}_{i j}= & -A_{R 2} \frac{p}{\mu} \frac{\sigma_{k\langle i} \sigma_{j\rangle k}}{\rho}+\left(\frac{8}{3} R T \sigma_{i j}-\frac{2}{7} R_{i j}\right) \frac{\partial u_{k}}{\partial x_{k}} \\
& -\frac{4}{7}\left(7 R T \sigma_{k\langle i}+R_{k\langle i}\right)\left(\frac{\partial u_{j\rangle}}{\partial x_{k}}+\frac{\partial u_{k}}{\partial x_{j\rangle}}\right) \\
& -2 R_{k\langle i} \frac{\partial u_{j\rangle}}{\partial x_{k}}+\frac{28}{5} \frac{q_{\langle i}}{\rho} \frac{\partial \sigma_{j\rangle k}}{\partial x_{k}}+\frac{28}{5} R T q_{\langle i}\left(\frac{\partial p}{p \partial x_{j\rangle}}-2 \frac{\partial T}{T \partial x_{j\rangle}}\right)+2 \frac{m_{i j k}}{\rho} \frac{\partial \sigma_{k l}}{\partial x_{l}} \\
& +m_{i j k}\left(\frac{2}{\rho} \frac{\partial p}{\partial x_{k}}-9 \frac{\partial R T}{\partial x_{k}}\right)+\frac{14}{3} \frac{\sigma_{i j}}{\rho}\left(\frac{\partial q_{m}}{\partial x_{m}}+\sigma_{m l} \frac{\partial u_{m}}{\partial x_{l}}\right) \\
& -\frac{14}{15} \Delta \frac{\partial u_{\langle i}}{\partial x_{j\rangle}}-2 \phi_{i j k l} \frac{\partial u_{k}}{\partial x_{l}},
\end{aligned}
$$




$$
\begin{aligned}
\aleph= & -A_{\Delta 2} \frac{p}{\mu} \frac{\sigma_{k j} \sigma_{j k}}{\rho}-\frac{4}{3} \Delta \frac{\partial u_{k}}{\partial x_{k}}-4\left(2 R T \sigma_{k l}+R_{k l}\right) \frac{\partial u_{k}}{\partial x_{l}}+8 \frac{q_{k}}{\rho}\left(\frac{\partial \sigma_{k l}}{\partial x_{l}}\right) \\
& +R T q_{k}\left(8 \frac{\partial p}{p \partial x_{k}}-28 \frac{\partial T}{T \partial x_{k}}\right) .
\end{aligned}
$$

\section{REFERENCES}

Acrivos, A., Leal, L. G., Snowden, D. D. \& Pan, F. 1968 Further experiments on steady separated flows past bluff objects. J. Fluid Mech. 34, 25-48.

Allen, M. P. \& Tildesley, D. J. 1987 Computer Simulation of Liquids. Clarendon Press.

APELT, C. J. 1961 The steady flow of a viscous fluid past a circular cylinder at Reynolds numbers 40 and 44. R.\&M. no. 3175. A.R.C. Technical Report. Her Majesty's Stationery Office.

Ashley, H. 1949 Applications of the theory of free molecule flow to aeronautics. J. Aeronaut. Sci. 16, 95-104.

Bairstow, L., CAVE, B. M. \& LANG, E. D. 1922 The two-dimensional slow motion of viscous fluids. Proc. R. Soc. Lond. A 100, 394-413.

BAKer, L. L. \& Hadjiconstantinou, N. G. 2005 Variance reduction for Monte Carlo solutions of the Boltzmann equation. Phys. Fluids 17, 051703.

BArber, R. W., Sun, Y., Gu, X. J. \& Emerson, D. R. 2004 Isothermal slip flow over curved surfaces. Vacuum 76 (1), 73-81.

BAsset, A. B. 1888 A Treatise on Hydrodynamics, vol. 2. George Bell and Sons.

BIRD, G. 1963 Approach to translational equilibrium in a rigid sphere gas. Phys. Fluids 6, 1518-1519.

BIRD, G. 1994 Molecular Gas Dynamics and the Direct Simulation of Gas Flows. Clarendon Press.

CANUto, D.\& TAIRA, K. 2015 Two-dimensional compressible viscous flow around a circular cylinder. J. Fluid Mech. 785, 349-371.

Cercignani, C. 1975 Theory and Application of the Boltzmann Equation. Scottish Academic Press.

Cercignani, C. 2000 Rarefied Gas Dynamics: From Basic Concepts to Actual Calculations. Cambridge University Press.

Chapman, S. \& Cowling, T. G. 1970 The Mathematical Theory of Non-uniform Gases. Cambridge University Press.

Coutanceau, M. \& Bouard, R. 1977 Experimental determination of the main features of the viscous flow in the wake of a circular cylinder in uniform translation. Part 1. Steady flow. J. Fluid Mech. 79, 231-256.

DenNis, S. C. R. \& CHANG, G.-Z. 1970 Numerical solutions for steady flow past a circular cylinder at Reynolds numbers up to 100. J. Fluid Mech. 40, 471.

Dennis, S. C. R. \& Shimshoni, M. 1965 The steady flow of a viscous fluid past a circular cylinder. C.P. No. 797. Her Majesty's Stationery Office.

Epstein, P. S. 1924 On the resistance experienced by spheres in their motion through gases. Phys. Rev. 23, 710-733.

Ferziger, J. H. \& Perić, M. 2002 Computational Methods for Fluid Dynamics, 3rd edn. Springer.

ForNBERG, B. 1980 A numerical study of steady viscous flow past a circular cylinder. J. Fluid Mech. 98, 819-855.

GAD-EL-HAK, M. 1999 The fluid mechanics of microdevices: the Freeman Scholar Lecture. J. Fluids Engng 121, 5-33.

Gallis, M. A., Bitter, N. P., Koehler, T. P., Torczynski, J. R., Plimpton, S. J. \& PAPAdAKIs, G. 2017 Molecular-level simulations of turbulence and its decay. Phys. Rev. Lett. 118, 064501.

Gallis, M. A., Koehler, T. P., Torczynski, J. R. \& Plimpton, S. J. 2016 Direct simulation Monte Carlo investigation of the Rayleigh-Taylor instability. Phys. Rev. Fluids 1, 043403.

Gallis, M. A., Torczynski, J. R., Plimpton, S. J., Rader, D. J. \& Koehler, T. P. 2014 Direct simulation Monte Carlo: the quest for speed. In 29th International Symposium on 
Rarefied Gas Dynamics (ed. J. Fan \& Q. Sun), AIP Conference Proceedings, vol. 1628, pp. 27-36. American Institute of Physics.

Grad, H. 1949 On the kinetic theory of rarefied gases. Commun. Pure Appl. Maths 2, 331-407.

Gu, X. J. \& Emerson, D. R. 2007 A computational strategy for the regularized 13 moment equations with enhanced wall-boundary conditions. J. Comput. Phys. 225, 263-283.

Gu, X. J. \& Emerson, D. R. 2009 A high-order moment approach for capturing non-equilibrium phenomena in the transition regime. J. Fluid Mech. 636, 177-216.

GU, X. J. \& EMERson, D. R. 2011 Modeling oscillatory flows in the transition regime using a high-order moment method. Microfluid. Nanofluid. 10, 389-401.

Gu, X. J. \& Emerson, D. R. 2014 Linearized-moment analysis of the temperature jump and temperature defect in the Knudsen layer of a rarefied gas. Phys. Rev. E 89, 063020.

Gu, X. J., EMERson, D. R. \& TANG, G. H. 2009 Kramers' problem and the Knudsen minimum: a theoretical analysis using a linearized 26-moment approach. Contin. Mech. Thermodyn. 21, 345.

Gu, X. J., Emerson, D. R. \& TAng, G. H. 2010 Analysis of the slip coefficient and defect velocity in the Knudsen layer of a rarefied gas using the linearized moment equations. Phys. Rev. E 81, 016313.

Gu, X. J., Zhang, H. \& Emerson, D. R. 2016 A new extended Reynolds equation for gas bearing lubrication based on the method of moments. Microfluid. Nanofluid. 20, 23.

HAPpel, J. \& BRENneR, H. 1983 Low Reynolds Number Hydrodynamics with Special Applications to Particulate Media. Martinus Nijhoff.

Heineman, M. 1948 Theory of drag in highly rarefied gases. Commun. Pure Appl. Maths 1, 259-273.

HIRSCH, C. 1991 Numerical Computation of Internal and External Flows II. Wiley.

HU, Y., SUN, Q. H. \& FAN, J. 2009 Simulation of gas flow over a micro cylinder. Proceedings of the ASME 2nd Micro/Nanoscale Heat \& Mass Transfer Inter. Conf., MNHMT2009-18288, Dec. 18-21, 2009, Shanghai, China.

Huner, B. \& Hussey, R. G. 1977 Cylinder drag at low Reynolds number. Phys. Fluids 20, $1211-1218$.

John, B., Gu, X. J., Barber, R. W. \& Emerson, D. R. 2016 High-speed rarefied flow past a rotating cylinder: the inverse Magnus effect. AIAA $J$ 54, 1670-1681.

John, B., GU, X. J. \& EMERSon, D. R. 2010 Investigation of heat and mass transfer in a lid-driven cavity under nonequilibrium flow conditions. Numer. Heat Transfer B 58, 287-303.

KAPLUN, S. 1957 Low Reynolds number flow past a circular cylinder. J. Math. Mech. 6, 595-603.

Kawaguti, M. \& Jain, P. 1966 Numerical study of a viscous fluid flow past a circular cylinder. J. Phys. Soc. Japan 21, 2055-2062.

Keller, J. B. \& WARD, M. J. 1996 Asymptotics beyond all orders for a low Reynolds number flow. J. Math. Mech. 30, 253-265.

Kumar, B. \& MitTAL, S. 2006 Prediction of the critical Reynolds number for flow past a circular cylinder. Comput. Meth. Appl. Mech. Engng 195, 6046-6058.

Lamb, H. 1911 On the uniform motion of a sphere through a viscous fluid. Phil. Mag. 21, 112-121.

Li, Z. H., Peng, A. P., Zhang, H. X. \& Deng, X. G. 2011 Numerical study on the gas-kinetic high-order schemes for solving Boltzmann model equation. Sci. China Phys. Mech. Astron. 54, 1687-1701.

Lockerby, D. A., Reese, J. M., Emerson, D. R. \& BArber, R. W. 2004 Velocity boundary condition at solid walls in rarefied gas calculations. Phys. Rev. E 70 (1), 017303.

Lu, Y. B., Tang, G. H., Sheng, Q., Gu, X. J., Emerson, D. R. \& Zhang, Y. H. 2017 Knudsen's permeability correction for gas flow in tight porous media using the R26 moment method. J. Porous Media 20, 787-805.

MAslach, G. I. \& SchaAF, S. A. 1963 Cylinder drag in the transition from continuum to freemolecular flow. Phys. Fluids 6, 315-321.

MaXwell, J. C. 1879 On stresses in rarified gases arising from inequalities of temperature. Phil. Trans. R. Soc. Lond. A 170, 231-256.

Muller, I. \& Ruggeri, T. 1993 Extended Thermodynamics. Springer. 
Munday, P. M., Taira, K., Suwa, T., Numata, D. \& Asai, K. 2015 Nonlinear lift on a triangular airfoil in low-Reynolds-number compressible flow. J. Aircraft 52, 924-931.

Oseen, C. W. 1910 Über die Stokessche Formel und über die verwandte Aufgabe in der Hydrodynamik. Ark. Mat. Astron. Fys. 6, 143-152.

PICH, J. 1969 The drag of cylinder in the transition region, 1969. J. Colloid Interface Sci. 29, 91-96.

Plimpton, S. J. \& Gallis, M. A. 2017 SPARTA Direct Simulation Monte Carlo (DSMC) Simulator. Sandia National Laboratories, USA, see http://sparta.sandia.gov.

Ponomarev, V. Y. \& Filippova, N. A. 1969 Experimental study of cylinder drag in a rarefied gas. Fluid Dyn. 4, 113-114.

Proudman, I. \& Pearson, J. R. A. 1957 Expansions at small Reynolds numbers for the flow past a sphere and a circular cylinder. J. Fluid Mech. 2, 237-262.

Rajani, B. N. A., Kandasamy, A. \& Majumdar, S. 2009 Numerical simulation of laminar flow past a circular cylinder. Appl. Math. Model. 33, 1228-1247.

SchaAf, S. A. \& Chambre, P. L. 1961 Flow of Rarefied Gases. Princeton University Press.

Sen, S., Mittal, S. \& Biswas, G. 2009 Steady separated flow past a circular cylinder at low Reynolds numbers. J. Fluid Mech. 620, 89-119.

Sentman, L. H. 1961 Free molecule flow theory and its application to the determination of aerodynamic forces, LMSC-448514, Lockheed Missiles \& Space Company.

Sheng, Q., TAng, G. H., Gu, X. J., Emerson, D. R. \& Zhang, Y. H. 2014 Simulation of thermal transpiration flow using a high-order moment method. Intl J. Mod. Phys. C 25, 1450061.

SKINNER, L. A. 1975 Generalized expansions for slow flow past a cylinder. Q. J. Mech. Appl. Maths 28, 333-340.

Son, J. S. \& HANRATTY, T. J. 1969 Numerical solution for the flow around a cylinder at Reynolds numbers of 40, 200 and 500. J. Fluid Mech. 35, 369-386.

Sone, Y. 2000 Flows induced by temperature fields in a rarefied gas and their ghost effect on the behavior of a gas in the continuum limit. Annu. Rev. Fluid Mech. 32, 779-811.

Stalder, J. R., Goodwin, G. \& Creager, M. O. 1951 A comparison of theory and experiment for high-speed free-molecule flow. NACA-TR-1032, National Advisory Committee for Aeronautics. Ames Aeronautical Lab., Moffett Field, CA.

Stokes, G. G. 1851 On the effect of the internal friction of fluids on the motion of pendulums. Trans. Camb. Phil. Soc. 9, 8-106.

Strouhal, V. 1878 Über eine besondere Art der Tonerregung. Ann. Phys. Chem. 5 (10), 216-251.

StruchtruP, H. 2005 Macroscopic Transport Equations for Rarefied Gas Flows. Springer.

Struchtrup, H. \& TORRILHON, M. 2003 Regularization of Grad's 13 moment equations: derivation and linear analysis. Phys. Fluids 15, 2668-2680.

Sun, Q. \& BOYD, I. D. 2004 Drag on a flat plate in low-Reynolds-number gas flows. AIAA $J \mathbf{4 2}$, 1066-1072.

TAheri, P. \& Struchtrup, H. 2009 Effects of rarefaction in microflows between coaxial cylinders. Phys. Rev. E 80, 066317.

TAheri, P. \& Struchtrup, H. 2010 An extended macroscopic transport model for rarefied gas flows in long capillaries with circular cross section. Phys. Fluids 22, 112004.

Taheri, P., Torrilhon, M. \& Struchtrup, H. 2009 Couette and Poiseuille microflows: analytical solutions for regularized 13-moment equations. Phys. Fluids 21, 017102.

TAKami, H. \& Keller, H. B. 1969 Steady two dimensional viscous flow of an incompressible fluid past a circular cylinder. Phys. Fluids 12, II-51.

TANEDA, S. 1956 Experimental investigation of the wakes behind cylinders and plates at low Reynolds numbers. J. Phys. Soc. Japan 11, 302-307.

Tang, G. H., Zhai, G. X., TAo, W. Q., Gu, X. J. \& Emerson, D. R. 2013 Extended thermodynamic approach for non-equilibrium gas flow. Commun. Comput. Phys. 13, 1330-1356.

Truesdell, C. \& Muncaster, R. G. 1980 Fundamentals of Maxwell's Kinetic Theory of a Simple Monotomic Gas. Academic Press.

TRItTON, D. J. 1959 Experiments on the flow past a circular cylinder at low Reynolds numbers. $J$. Fluid Mech. 6, 547-567. 
Tritton, D. J. 1988 Physical Fluid Dynamics. Oxford University Press.

TSIEN, H.-S. 1946 Superaerodynamics, mechanics of rarefied gases. J. Aeronaut Sci. 13, 653-664.

UNDERWOOD, R. L. 1969 Calculation of incompressible flow past a circular cylinder at moderate Reynolds numbers. J. Fluid Mech. 37, 95-114.

Volkov, A. N. \& Sharipov, F. 2017 Flow of a monatomic rarefied gas over a circular cylinder: calculations based on the ab initio potential method. Intl J. Heat Mass Transfer 114, 47-61.

Westerkamp, A.\& Torrilhon, M. 2012 Slow rarefied gas flow past a cylinder: analytical solution in comparison to the sphere. AIP Conf. Proc. 1501 (1), 207-214.

White, F. M. 1991 Viscous Fluid Flow. McGraw-Hill.

Wu, L., Ho, M. T., Germanou, L., Gu, X. J., LiU, C., Xu, K. \& Zhang, Y. H. 2017 On the apparent permeability of porous media in rarefied gas flows. J. Fluid Mech. 822, 398-417.

Wu, M.-H., Wen, C.-Y., Yen, R.-H., Weng, M.-C. \& WANG, A.-B. 2004 Experimental and numerical study of the separation angle for flow around a circular cylinder at low Reynolds number. J. Fluid Mech. 515, 233-260.

Yamamoto, K. \& Sera, K. 1985 Flow of a rarefied gas past a circular cylinder. Phys. Fluids 28, $1286-1293$

Young, J. B. 2011 Calculation of Knudsen layers and jump conditions using the linearised G13 and R13 moment methods. Intl J. Heat Mass Transfer 54, 2902-2912.

Zdravkovich, M. 1997 Flow around Circular Cylinders, vol. 1. Oxford Science. 